\title{
Reproducibility of Parallel \\ Preconditioned Conjugate Gradient in \\ Hybrid Programming Environments
}

\author{
Roman lakymchuk ${ }^{1,2}$, Maria Barreda ${ }^{3}$, Stef Graillat ${ }^{1}$, José I. Aliaga ${ }^{3}$, Enrique S. \\ Quintana-Ortí ${ }^{4}$
}

\begin{abstract}
The Preconditioned Conjugate Gradient method is often employed for the solution of linear systems of equations arising in numerical simulations of physical phenomena. While being widely used, the solver is also known for its lack of accuracy while computing the residual. In this article, we propose two algorithmic solutions that originate from the ExBLAS project to enhance the accuracy of the solver as well as to ensure its reproducibility in a hybrid MPI + OpenMP tasks programming environment. One is based on ExBLAS and preserves every bit of information until the final rounding, while the other relies upon floating-point expansions and, hence, expands the intermediate precision. Instead of converting the entire solver into its ExBLAS-related implementation, we identify those parts that violate reproducibility/non-associativity, secure them, and combine this with the sequential executions. These algorithmic strategies are reinforced with programmability suggestions to assure deterministic executions. Finally, we verify these approaches on two modern HPC systems: both versions deliver reproducible number of iterations, residuals, direct errors, and vector-solutions for the overhead of less than $37.7 \%$ on 768 cores.
\end{abstract}

\section{Keywords}

Preconditioned Conjugate Gradient, MPI, OpenMP tasks, reproducibility, accuracy, floating-point expansion, long accumulator, fused multiply-add.

\section{Introduction}

Many current scientific and engineering problems involve the solution of large and sparse linear systems of equations. Some traditional examples appear, for example, in circuit and device simulation, quantum physics, large-scale eigenvalue computations, nonlinear sparse equations, and all sorts of applications that include the discretization of partial differential equations (PDEs) Barrett et al. (1994). For many problems (especially those associated with 3-D models), the size and complexity of these systems have turned iterative projection methods, based on Krylov subspaces, into a highly competitive approach compared with direct solvers Saad (2003). In particular, the Conjugate Gradient (CG) method is one of the most efficient Krylov subspacebased algorithms for the solution of sparse linear systems when the coefficient matrix is symmetric positive definite (s.p.d.) Saad (2003). Preconditioning is usually incorporated in real implementations of the method in order to accelerate the convergence of the method and improve its numerical features, yielding the Preconditioned Conjugate Gradient (PCG) method.

One would expect that the results of different runs of PCG are identical, for instance, in the number of iterations, the intermediate and final residuals, as well as the solutionvector. However, in practice this is not often the case due to different reduction trees - the Message Passing Interface (MPI) implementations (libraries) Gropp et al. (2014) offer up to 14 different implementations for reduction -, OpenMP tasks scheduling, data alignment, instructions used, etc. Each of these factors may change the execution order of floatingpoint operations, which are commutative but non-associative, and, hence, result in non-reproducible results. We define reproducibility as the ability to obtain a bit-wise identical and accurate result for multiple executions on the same data. Therefore, our aim of this study is to ensure reliable computations (we also refer to them as robust), at reasonable cost, for codes that leverage PCG (or any similar Krylov subspace solver) and encounter numerical issues during sensitive computations of the residual. Our approach and routines are also aimed to be used for debugging as we ensure reproducible residuals, direct errors, number of iterations, and solution-vector from the sequential, pure MPI, and even hybrid MPI + OpenMP versions.

Ensuring the bit-wise reproducibility is often a complex and expensive task that imposes modifications to the algorithm and its underlying parts such as the BLAS (Basic Linear Algebra Subprograms) routines Lawson et al. (1979); Dongarra et al. (1990). These modifications are necessary to preserve every bit of information (both result and error) Collange et al. (2015) or, alternatively, to cut off some parts of the data and operate on the remaining most-significant parts Mukunoki et al. (2020); Demmel and Nguyen (2015). Furthermore, the bit-wise

\footnotetext{
${ }^{1}$ Sorbonne Université, LIP6, France

${ }^{2}$ Fraunhofer ITWM, Germany

${ }^{3}$ Universitat Jaime I, Spain

${ }^{4}$ Universitat Politècnica de València, Spain
}

\section{Corresponding author:}

Roman lakymchuk, Sorbonne Université, LIP6, Campus Pierre et Marie Curie, 4 place Jussieu, 75252 PARIS CEDEX 05, France

Email: roman.iakymchuk@sorbonne-universite.fr 
reproducibility can become expensive with the overhead of at least $8 \%$ for parallel reduction Collange et al. (2015); Demmel and Nguyen (2015), up to $2 \mathrm{x}-4 \mathrm{x}$ for matrix-vector product Iakymchuk et al. (2019b), and more than 10x for matrix-matrix multiplication Iakymchuk et al. (2016). In this paper, we aim to revisit reproducibility and raise its appeal through reducing its negative impact on performance and minimizing changes to both the algorithm and its building blocks. We also raise a question: Can reproducibility of algorithms be ensured by design with both minimal changes to algorithms and almost negligible overhead? Hence, our idea is to address those parts of algorithms that violate associativity - such as parallel reductions, dot products, and possible replacements by compilers of $a * b+c$ in favor of fused multiply-add (fma) operation, etc. - as well as to combine that with sequential executions of subblocks/subroutines. Such sequential execution of operations is reproducible under some constraints, for example the same initial conditions on the input data like data alignment.

We consider to verify this idea (both algorithmic and programmability) on a typical sparse linear algebra solver such as PCG and ensure its reproducibility on parallel distributed-memory systems using a hybrid combination of the MPI + OpenMP-tasks programming models. On one hand, the hybridization reduces the communication burden being more focused on inner node computations and work balancing, especially on nodes with large core counts such as those in the MareNostrum4 platform at Barcelona Supercomputing Center. On the other hand, it introduces a new challenge in the form of a double-level reduction: an initial reduction among tasks inside a process/node, followed by one among processes. Thus, we ensure reproducibility of the PCG solver by preventing non-deterministic executions as follows:

- We construct two reproducible solutions: a first one on the ExBLAS approach Iakymchuk et al. (2017) and an alternative lightweight version based on floating-point expansions (FPEs). The ExBLAS-based approach with its cornerstone Kulisch long accumulator Kulisch (2013) is robust but expensive since it is designed to cover severe (ill-conditioned) cases with very broad dynamic ranges. Motivated by " 100 bits suffice for many HPC applications" as noted by David Bailey at ARITH-21 Bailey (2013) and a mini accumulator from the ARM team Lutz and Hinds (2017); Burgess et al. (2019), we derive a faster but less generic version using FPEs, which is the other core algorithmic component in the ExBLAS approach, aiming to adjust the algorithm to the problem at hand.

- As a consequence, we also address the common issue of sparse iterative solvers - the accuracy while computing the residual - and propose to use solutions that offer reproducibility (and potentially correctrounding) only while computing the corresponding dot products.

- Hence, we derive two hybrid (MPI + OpenMP tasks), reproducible, and accurate dot products using ExBLAS and FPEs.

- Finally, we demonstrate applicability and feasibility of the aforementioned idea with the ExBLAS- and FPE-based approaches in the hybrid MPI + OpenMP implementation of PCG on an example of a 3D Poisson's equation with 27 stencil points as well as several test matrices from the SuiteSparse matrix collection. This extends our previous results with the pure MPI implementation of PGC Iakymchuk et al. (2019a) to the more complex double-level dot products and reductions with dynamic scheduling of the tasks.

To sum up: the FPE-based (we also call it Opt) solution is efficient and fast, but it is limited to cases where the condition number and/or the dynamic range do not exceed certain thresholds, e.g. the dynamic range is below $10^{50}$. (At this point, we note that the condition of a linear system can be cheaply estimated with fair accuracy.) In comparison, the ExBLAS-based solution is reserved for extreme cases as well as problems where we do not have any information about the problem at hand.

This article is organized as follows. Section 2 reviews several aspects of computer arithmetic, in particular floatingpoint expansion and long accumulator, as well as the ExBLAS approach for accurate and reproducible computations. Section 3 introduces the PCG algorithms and describes in details its hybrid (MPI+OpenMP) implementation. We present strategies for ensuring reproducibility of PCG in Section 4 and evaluate corresponding implementations in Section 5. Finally, Section 6 reviews related work, while Section 7 draws conclusions and outlines future directions.

\section{Background}

At first, we briefly introduce the floating-point arithmetic that consists in approximating real numbers by numbers that have a finite, fixed-precision representation. These numbers are composed of a significand, an exponent, and a sign: $x=$ $\pm \underbrace{x_{0} \cdot x_{1} \ldots x_{M-1}}_{\text {mantiss }} \times b^{e}, 0 \leq x_{i} \leq b-1, x_{0} \neq 0$, where $b$ is the basis ( 2 in our case), $M$ is the precision, and $e$ stands for the exponent that is bounded $\left(e_{\min } \leq e \leq e_{\max }\right)$.

The IEEE 754 standard IEEE Computer Society (2008), created in 1985 and then revised in 2008, has led to a considerable enhancement in the reliability of numerical computations by rigorously specifying the properties of floating-point arithmetic. This standard is now adopted by most processors, thus leading to a much better portability of numerical applications. The standard specifies floatingpoint formats, which are often associated with precisions like binary16, binary32, and binary64, see Table 1. Floatingpoint representation allows numbers to cover a wide dynamic range that is defined as the absolute ratio between the number with the largest magnitude and the number with the smallest non-zero magnitude in a set. For instance, binary 64 (double-precision) can represent positive numbers from $4.9 \times 10^{-324}$ to $1.8 \times 10^{308}$, so it covers a dynamic range of $3.7 \times 10^{631}$

The IEEE 754 standard requires correctly rounded results for the basic arithmetic operations $(+,-, \times, /, \sqrt{ }$, fma). It means that the operations are performed as if the result was first computed with an infinite precision and then rounded to the floating-point format. The correct rounding criterion guarantees a unique, well-defined answer, ensuring bit-wise reproducibility for a single operation. Several rounding modes are provided. The standard also contains the 
Table 1. Parameters for three IEEE arithmetic precisions; quadruple (128 bits) is omitted.

\begin{tabular}{llllll}
\hline Type & Size & Significand & Exponent & Rounding unit & Range \\
\hline half & 16 bits & 11 bits & 5 bits & $u=2^{-11} \approx 4.88 \times 10^{-4}$ & $\approx 10^{ \pm 5}$ \\
single & 32 bits & 24 bits & 8 bits & $u=2^{-24} \approx 5.96 \times 10^{-8}$ & $\approx 10^{ \pm 38}$ \\
double & 64 bits & 53 bits & 11 bits & $u=2^{-53} \approx 1.11 \times 10^{-16}$ & $\approx 10^{ \pm 308}$ \\
\hline
\end{tabular}

reproducibility clause that forwards the reproducibility issue to language standards. Emerging attention to reproducibility strives to draw more careful attention to the problem by the computer arithmetic community. It has led to the inclusion of error-free transformations (EFTs) for addition and multiplication - to return the exact outcome as the result and the error - to assure numerical reproducibility of floatingpoint operations, into the revised version of the standard. These mechanisms, once implemented in hardware, will simplify our reproducible algorithms - like the ones used in the ExBLAS Collange et al. (2015), ReproBLAS Demmel and Nguyen (2015), OzBLAS Mukunoki et al. (2020) libraries - and boost their performance.

There are three approaches that enable the addition of floating-point numbers without incurring round-off errors or with reducing their impact. The main idea is to keep track of both the result and the errors during the course of computations. The first approach uses EFT to compute both the result and the rounding error and stores them in a floating-point expansion (FPE), which is an unevaluated sum of $p$ floating-point numbers, whose components are ordered in magnitude with minimal overlap to cover the whole range of exponents. Typically, FPE relies upon the use of the traditional EFT for addition that is twosum Knuth (1969) (Algorithm 1) and for multiplication that is twoprod EFT Ogita et al. (2005) (Algorithm 2). Note that the underlying architecture should support fma, which is often the case. Otherwise, we refer to Algorithm 3.3 in Ogita et al. (2005), which relies on Dekker's algorithm for splitting a floatingpoint number Dekker (1971); this altogether requires 17 flops in contrary to 3 flops of Algorithm 2 with fma.
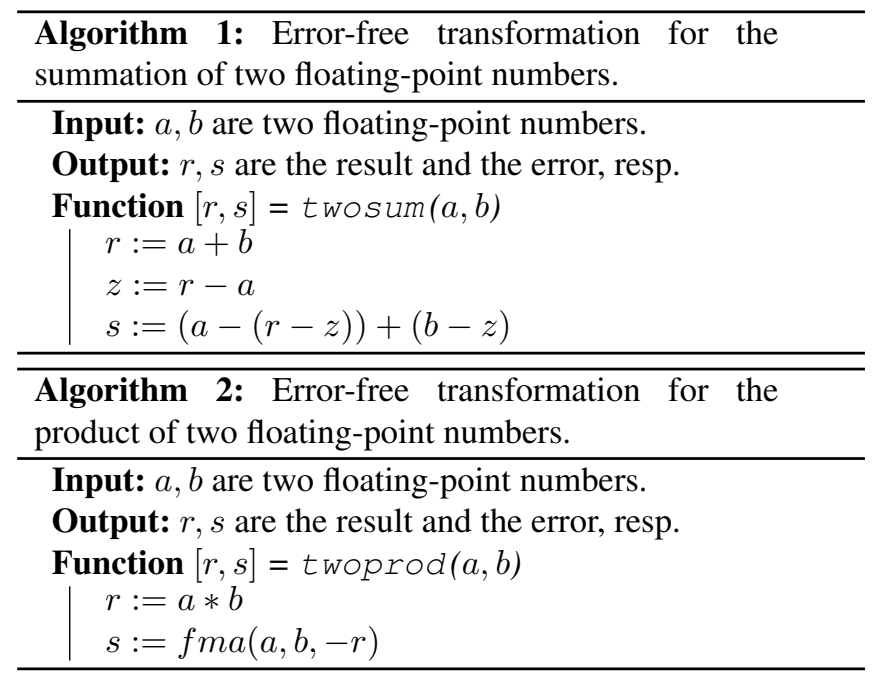

The second approach projects the finite range of exponents of floating-point numbers into a long vector so called a long (fixed-point) accumulator and stores every bit there. For instance, Kulisch Kulisch and Snyder (2011) proposed to use a 4288-bit long accumulator for the exact dot product of two vectors composed of bi nary 64 numbers; such a large long accumulator is designed to cover all the severe cases without overflows in its highest digit.

The third approach is based on slicing or splitting a floating-point number into slices using Dekker's algorithm. Then, the same work is carried separately on each slice and the accumulated results are aggregated/merged. More details and analysis can be found in Rump et al. (2008a), with ideas originating from Zielke and Drygalla (2003). This approach is implemented in ReproBLAS and OzBLAS.

\subsection{ExBLAS - Exact BLAS}

The ExBLAS project Iakymchuk et al. (2015) is an effort to derive fast, accurate, and reproducible BLAS library by constructing a multi-level approach for these operations that are tailored for various modern architectures with their complex multi-level memory structures. On one side, this approach aims to ensure similar performance compared with the non-deterministic parallel counterparts. On the other side, the approach preserves every bit of information before the final rounding to the desired format to assure correct-rounding and, therefore, reproducibility. Hence, ExBLAS combines together long accumulator and FPE into algorithmic solutions. In addition, it efficiently tunes and implements them on various architectures, including conventional CPUs, NVIDIA and AMD GPUs, and Intel Xeon Phi co-processors (for details we refer to Collange et al. (2015)). Thus, ExBLAS assures reproducibility through assuring correct-rounding.

The cornerstone of ExBLAS is the reproducible parallel reduction, which is at the core of many BLAS routines. The ExBLAS parallel reduction relies upon FPEs with the twosum EFT Knuth (1969) and long accumulators, so it is correctly rounded and reproducible. In practice, the latter is invoked only once per overall summation which results in the little overhead (less than $8 \%$ ) on accumulating large vectors. Our interest in this article is the dot product of two vectors, which is another crucial fundamental BLAS operation. The EXDOT algorithm is based on the previous EXSUM algorithm and the twoprod EFT Ogita et al. (2005) (see Algorithm 2): the algorithm accumulates the result and the error of twoprod to same FPEs and then follows the EXSUM scheme. These and the other routines - such as matrix-vector product, triangular solve, and matrix-matrix multiplication - are distributed in the ExBLAS library*. In this paper, we derive a hybrid MPI + OpenMP tasks EXDOT, where a long accumulator is shared among OpenMP tasks within one process and each OpenMP thread owns two FPEs underneath (one for the result and the other for the error) that are merged at the end of computations .

*ExBLAS repository: https://github. com/riakymch/exblas. 


\section{Algorithm(s)}

In this section we review the PCG algorithm and its taskparallel implementation using MPI and OpenMP tasks. The goal of the following analysis is twofold: to offer a complete description of the parallelization approach and, even more important, to identify key inter-node (i.e., between MPI ranks) and intra-node (i.e., between threads executing tasks) communications, in particular reductions, which pose a challenge to ensuring reproducibility.

\subsection{Preconditioned Conjugate Gradient Solver}

We consider the linear system $A x=b$, where the coefficient matrix $A \in \mathbb{R}^{n \times n}$ is sparse and symmetric positive definite (s.p.d.), with $n_{z}$ nonzero entries; $b \in \mathbb{R}^{n}$ is the righthand side vector; and $x \in \mathbb{R}^{n}$ is the sought-after solution vector. Figure 1 presents the algorithmic description of the classical iterative PCG. In the body loop of the algorithm, the following operations are executed: a sparse matrixvector product (SPMV) (S1), three DOT products (S2,S6, and S8), three AXPY (-like) operations (S3, S4, and $\mathrm{S} 7)$, the preconditioner application (S5), and a few scalar operations Barrett et al. (1994). In particular, in the proposed implementation of the PCG method, we incorporate a Jacobi preconditioner Saad (2003), which is composed of the elements in the diagonal of the matrix $(M:=D=$ $\operatorname{diag}(A)$ ). Therefore, the application of the preconditioner is carried out on a vector and involves an element-wise multiplication of two vectors.

\subsection{Message-passing PCG}

In this subsection we analyse the communication patterns of a message-passing implementation of the PCG solver that operates in a distributed-memory platform. For clarity, hereafter we will drop the superindices that denote the iteration count in the variable names. The following considerations are taken into account in the analysis of the communications:

- The parallel platform comprises $K$ processes (or MPI ranks), denoted as $P_{1}, P_{2}, \ldots, P_{K}$.

- The coefficient matrix $A$ is partitioned into $K$ blocks of rows $\left(A_{1}, A_{2}, \ldots, A_{k}\right)$, with the $k$-th distribution block $A_{k} \in \mathbb{R}^{p_{k} \times n}$ stored in $P_{k}$, and $n=\sum_{k=1}^{K} p_{k}$.

- Vectors are partitioned and allocated conformally with the block-row distribution of $A$. For example, the residual vector $r$ is partitioned as $r_{1}, r_{2}, \ldots, r_{K}$, where $P_{k}$ stores $r_{k}$

- The scalars $\alpha, \beta, \rho, \tau$ are replicated on all $K$ processes.

Considering these previous aspects, we next examine how they affect the different computational kernels $(S 1-S 8)$ that are executed in a single PCG iteration in Figure 1.

Sparse matrix-vector product (S1): The input operands are the coefficient matrix $A$, which is distributed by blocks of rows, and the vector $d$, which is partitioned and distributed according to $A$. A communication stage is required before executing this kernel in order to assemble the distributed parts of vector $d$ into a single vector $e$, which is replicated in all processes. We denote this communication as $d \rightarrow e$, which can be performed in MPI via an MPI_Allgatherv. Note that vector $e$ is the only array that is replicated in all processes. After that, the computation can proceed in parallel and each process calculates its local slice of the output vector $w: P_{k}: w_{k}:=A_{k} e$.

DOT products $(S 2, S 6, S 8)$ : In this kernel, each process can compute concurrently a partial result (in step $S 2$, $P_{k}$ calculates $\rho_{k}:=<d_{k}, w_{k}>$ ). Then, these intermediate values are reduced into a globally-replicated scalar (for example, $\rho:=\beta /\left(\rho_{1}+\rho_{2}+\cdots+\rho_{K}\right)$ in $S 2$ ). We implement this reduction in MPI using MPI_Allreduce. Applying this idea to all the DOT products, there are three process synchronizations because $\rho, \beta, \tau$ are globallyreplicated.

AXPY(-type) vector updates (S3, S4, S7): The AXPY kernel involves two distributed vectors ( $x$ and $d$ in $S 3$ ) and a globally-replicated scalar ( $\rho$ in S3). This kernel can be executed concurrently because all processes can perform their local parts of the computation without any communication $\left(P_{k}: x_{k}:=x_{k}+\rho d_{k}\right)$.

Application of the preconditioner (S5): The kernel in step $S 5$ consists in applying the Jacobi preconditioner $M$. In order to do that, vector $r$ is scaled by the diagonal of the matrix. Here, each process stores a different group of the diagonal elements and also a local piece of the vector $r$, so that, the computations can be done in parallel, i.e $P_{k}: z_{k}:=M_{k}^{-1} r_{k}$.

The algorithm with communications is summarized in Figure 2. We can re-arrange the operations to reduce the the number of synchronizations in the loop body of the PCG solver, as shown there. Concretely, pushing up step $S 8$ next to step $S 6$, we can simultaneously execute these two reductions by merging them into one reduction and, hence, the number of synchronizations decreases from three to two per iteration of PCG.

\subsection{Task-parallelism in message-passing PCG}

In a cluster of multicore processors, a good practice to increase the performance of the codes is to introduce an additional level of parallelism. This level is exploited in each node of the cluster using, for example, OpenMP. The analysis in Aliaga et al. (2017); Barreda et al. (2019) exposes that, in the PCG, a reasonable option is to leverage task-parallelism, which consists in dividing each kernel into a collection of finer-grain operations, or tasks. Then, each thread executes a different task and two consecutive kernels can be executed concurrently avoiding a thread-synchronization point after each kernel, as described next.

In the following analysis, for simplicity, we merge the execution of $S 3$ with that of $S 4$; and $S 8$ with $S 6$. Therefore, we will only consider kernels $S 1-S 2$ and $S 4-S 7$ in the loop body of the PCG solver (see Figure 2). Thus, the operations in the solver are interlaced by a series of data dependencies which impose a strict order of execution:

$$
\underbrace{\cdots S 7}_{\text {iteration } l-1} \stackrel{d}{\rightarrow} S 1 \stackrel{w}{\rightarrow} S 2 \stackrel{\rho}{\rightarrow} S 4 \stackrel{r}{\rightarrow} S 5 \stackrel{z}{\rightarrow} S 6 \stackrel{\beta}{\rightarrow} S 7 \stackrel{d}{\rightarrow} \underbrace{S 1 \cdots}_{\text {iteration } l} \underbrace{}_{\text {iteration } l+1},
$$

where the variable that generates the dependency is denoted on top of each dependency arrow.

Exploiting task-parallelism allows that some of these kernels can be (partially) computed concurrently (denoted with the symbol "||"), breaking the strict inter-kernel barriers due to the dependencies; in particular, we aim to attain a parallel execution with $S 1 \| S 2$ and $S 4\|S 5\| S 6$. 


\begin{tabular}{|c|c|c|}
\hline \multicolumn{3}{|c|}{$\begin{array}{l}\text { Compute preconditioner for } A \rightarrow M \\
\text { Set starting guess } x^{(0)} \\
\text { Initialize } z^{(0)}, d^{(0)}, \beta^{(0)}, \tau^{(0)}, l:=0 \text { (iteration count) }\end{array}$} \\
\hline \multicolumn{3}{|c|}{$\begin{array}{l}r^{(0)}:=b-A x^{(0)} \\
\tau^{0}:=<r^{(0)}, r^{(0)}> \\
\text { while }\left(\tau^{(l)}>\tau_{\max }\right)\end{array}$} \\
\hline Step & Operation & Kernel \\
\hline S1: & $w^{(l)} \quad:=A d^{(l)}$ & SPMV \\
\hline S2 : & $\rho^{(l)} \quad:=\beta^{(l)} /<d^{(l)}, w^{(l)}>$ & DOT product \\
\hline S3: & $x^{(l+1)}:=x^{(l)}+\rho^{(l)} d^{(l)}$ & AXPY \\
\hline$S_{4}:$ & $r^{(l+1)}:=r^{(l)}-\rho^{(l)} w^{(l)}$ & AXPY \\
\hline S5 : & $z^{(l+1)}:=M^{-1} r^{(l+1)}$ & Apply precond. \\
\hline$S 6:$ & $\beta^{(l+1)}:=\left\langle z^{(l+1)}, r^{(l+1)}>\right.$ & DOT product \\
\hline$S 7:$ & $d^{(l+1)}:=\left(\beta^{(l+1)} / \beta^{(l)}\right) d^{(l)}+z^{(l+1)}$ & AXPY-like \\
\hline S8: & $\begin{aligned} \tau^{(l+1)} & :=<r^{(l+1)}, r^{(l+1)}> \\
l & :=l+1\end{aligned}$ & DOT product \\
\hline \multicolumn{3}{|c|}{ end while } \\
\hline
\end{tabular}

Figure 1. Formulation of the PCG solver annotated with computational kernels. The threshold $\tau_{\max }$ is an upper bound on the relative residual for the computed approximation to the solution. In the notation, $\langle\cdot, \cdot\rangle$ computes the DOT (inner) product of its vector arguments.

Sparse matrix-vector product S1 || DOT product S2: On the one hand, the local operands to process $P_{k}$ of the SPMV can be divided as $w_{k}:=A_{k}$ e:

$$
\left[\begin{array}{c}
\tilde{w}_{1} \\
\hline \tilde{w}_{2} \\
\hline \vdots \\
\hline \tilde{w}_{I}
\end{array}\right]:=\left[\begin{array}{c|c|c|c}
\tilde{A}_{1,1} & \tilde{A}_{1,2} & \ldots & \tilde{A}_{1, J} \\
\hline \tilde{A}_{2,1} & \tilde{A}_{2,2} & \ldots & \tilde{A}_{2, J} \\
\hline \vdots & \vdots & \ddots & \vdots \\
\hline \tilde{A}_{I, 1} & \tilde{A}_{I, 2} & \ldots & \tilde{A}_{I, J}
\end{array}\right]\left[\begin{array}{c}
e_{1} \\
\hline e_{2} \\
\hline \ddots \\
\hline e_{J}
\end{array}\right] .
$$

Here, we can consider each group of rows as a task, which computes the corresponding SPMV operation to obtain a partial result, $\tilde{w}_{k}$. For example, if we consider $\tilde{w}_{1}$, there is a task calculating $\tilde{w}_{1}=\sum_{j=1}^{J} \tilde{A}_{1, j} e_{j}$.

On the other hand, the computation local to $P_{k}$ for the DOT product $S 2$ can be decomposed into $S$ tasks. These tasks can be computed concurrently by partitioning the input operands $d_{k}, w_{k}$ into $S$ pieces, with each task obtaining a partial result $\tilde{\rho}_{s}$ :

$\rho_{k}:=<d_{k}, w_{k}>\equiv \tilde{\rho}_{s}:=<\tilde{d}_{s}, \tilde{w}_{s}>, s=1,2, \ldots, S$.

These partial results are reduced to generate a unique value local to the $k$-th node, $\rho_{k}:=\sum_{s=1}^{S} \tilde{\rho}_{s}$, and these local values are thereafter reduced across all $K$ nodes to produce the globally replicated scalar $\rho:=\sum_{k=1}^{K} \rho_{k}$.

Note that the advantage here is that we can eliminate the dependency $S 1 \rightarrow S 2$, by splitting up these operations into fine-grain tasks. Hence, the execution of some tasks of the second kernel can start as soon as the corresponding results of the previous one are available, resulting in a partiallyparallel execution of these two tasks. However, the global reduction required at the end of $S 2$ enforces a task/process synchronization point that is an impediment to extend this idea further that point.

AXPY vector update $S 4 \|$ preconditioner application $S 5$ \| DOT product S6: $S 4-S 6$ can be computed in parallel by

\begin{tabular}{|c|c|c|}
\hline \multicolumn{3}{|c|}{$\begin{array}{l}\text { Compute preconditioner for } A \rightarrow M \\
\text { Set starting guess } x \\
\text { Initialize } z, d, \beta, \tau, l:=0\end{array}$} \\
\hline \multicolumn{3}{|c|}{$\begin{array}{l}r:=b-A x \\
\tau:=<r, r> \\
\text { while }\left(\tau>\tau_{\max }\right)\end{array}$} \\
\hline Step & Operation & Communication \\
\hline & $\beta^{\prime}:=\beta$ & - \\
\hline $\begin{array}{l}\text { S1: } \\
\text { S1.1: } \\
\text { S1.2: }\end{array}$ & $\begin{array}{l}d \rightarrow e \\
w:=A e\end{array}$ & $\begin{array}{l}\text { Allgatherv } \\
-\end{array}$ \\
\hline S2: & $\rho:=\beta /<d, w>$ & Allreduce \\
\hline $\begin{array}{l}\text { S3: } \\
\text { S4: } \\
\text { S5: }\end{array}$ & $\begin{aligned} x & :=x+\rho d \\
r & :=r-\rho w \\
z & :=M^{-1} r\end{aligned}$ & $\begin{array}{l}- \\
- \\
-\end{array}$ \\
\hline $\begin{array}{l}S 6: \\
S 8:\end{array}$ & $\begin{aligned} \beta & :=<z, r> \\
\tau & :=<r, r>\end{aligned}$ & $\begin{array}{l}\text { Allreduce } \\
\text { Allreduce }\end{array}$ \\
\hline $\begin{array}{l}S 7 \text { : } \\
\text { end while }\end{array}$ & $\begin{aligned} d & :=\left(\beta / \beta^{\prime}\right) d+z \\
l & :=l+1\end{aligned}$ & - \\
\hline
\end{tabular}

Figure 2. Message-passing formulation of the PCG solver annotated with communication. applying a similar division of the three kernels into finegrain. Nevertheless, again a task/process synchronization is required right after $S 6$.

AXPY vector update S7 and SPMV S1 (subsequent iteration): The convergence test and the requirement to perform the replication $d \rightarrow e$ at the beginning of each iteration, inserts a process synchronization that makes impossible the concurrent computation of the local tasks corresponding to these two kernels.

\subsection{Implementation using MPI+OpenMP}

In this subsection we detail how to exploit the described two levels of parallelism via a combination of two parallel programming interfaces: MPI forum (2019) and OpenMP OpenMP ARB (2019).

We leverage OpenMP tasks to implement task-parallelism. At execution time, the runtime system underlying OpenMP detects data dependencies between tasks, with the help of compiler directives (\#pragma omp task) annotated with clauses that indicate the task operands' directionality (input (in), output (out) or both (inout)). Then, a task graph is generated during the execution, which is used to schedule the tasks to the cores, exploiting the inherent task-level parallelism while fulfilling the dependencies embedded in the graph.

As an example, the DOT product, which computes $\alpha:=$ $x^{T} y, x, y \in \mathbb{R}^{q}$, is annotated as

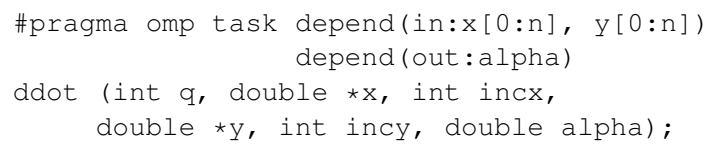

For the routine AXPY $y:=y+\alpha x$, the code snippet using OpenMP tasks is as follows:

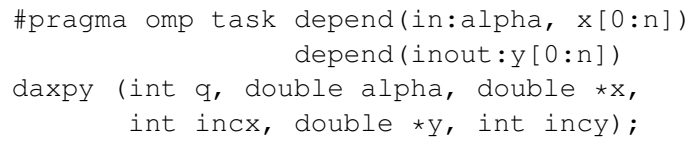


The replication of vector $d$ into $e$ is performed across the processes using the MPI collective MPI_Allgatherv, as stated previously. To ensure that all the processes have finalized their computation of $d$ prior to the MPI collective, we introduce a task barrier, using the directive \#pragma omp taskwait. This creates a task synchronization point because it enforces that all tasks up to that point are completed. Furthermore, this syncronization point is leveraged to perform the convergence test $\left(\tau>\tau_{\max }\right.$ ?) right after it, which is followed by an implicit MPI syncronization across processes in the MPI collective primitive.

The MPI_Al lreduce primitive is used to implement the global reductions. Similarly to the previous case, we insert a \#pragma omp taskwait on the specific variable being reduced before invoking the MPI collectives for the reduction. This ensures that all tasks operating on that variable have been finalized prior the reduction across nodes can start. Moreover, atomic updates are employed to accumulate the results from each reduction task (e.g., $\widetilde{\beta}_{n}$ for $S 8)$ into the local result $\left(\beta_{k}\right)$.

The previous description is condensed in Figure 3, focusing on the operations computed by the process $P_{k}$, during the iteration $l$.

\section{Reproducibility of PCG}

In this section, we present our strategies for ensuring reproducibility of the PCG solver. The first strategy relies on the ExBLAS approach, while the second is derived from it and is based on FPEs. Both strategies are reinforced with programmability components such as the explicit use of fma instructions and a careful re-arrangement of computations. Therefore, the reproducibility of the PCG solver is guaranteed via reproducibility of its building blocks on each iteration.

\subsection{ExBLAS-based Strategy}

Section 2.1 provides an overview of the ExBLAS approach. Here we exploit the ExBLAS parallel reduction in conjunction with the twoprod EFT to derive a hybrid MPI (inter-node, distributed) and OpenMP (intra-node) for the DOT products appearing in PCG. The intra-node DOT product is presented here, and its distributed part is described in Section 4.3 together with the FPE-based alternative.

For accurate and reproducible DOT product within an MPI process, we rely upon OpenMP tasks following the ExBLAS ${ }_{6}^{5}$ approach. We allocate one long accumulator per MPI process as well as, within the exblas: :cpu: :exdot, a vector of FPEs shared among OpenMP threads. Hence, the work ${ }_{9}^{8}$ on the process-local DOT products is divided into multiple ${ }^{10}$ task (more than threads) in such a way that the intermediate ${ }_{12}^{11}$ results from each task are stored in this large vector of 13 FPEs. To complete the local DOT products we flush all ${ }_{15}^{14}$ FPEs sequentially into the process-owned long accumulator. 16 Listing 1 outlines the code snippet of this implementation. Accumulate is presented in Algorithm 4, however here it also includes a possibility to flush the error to the long

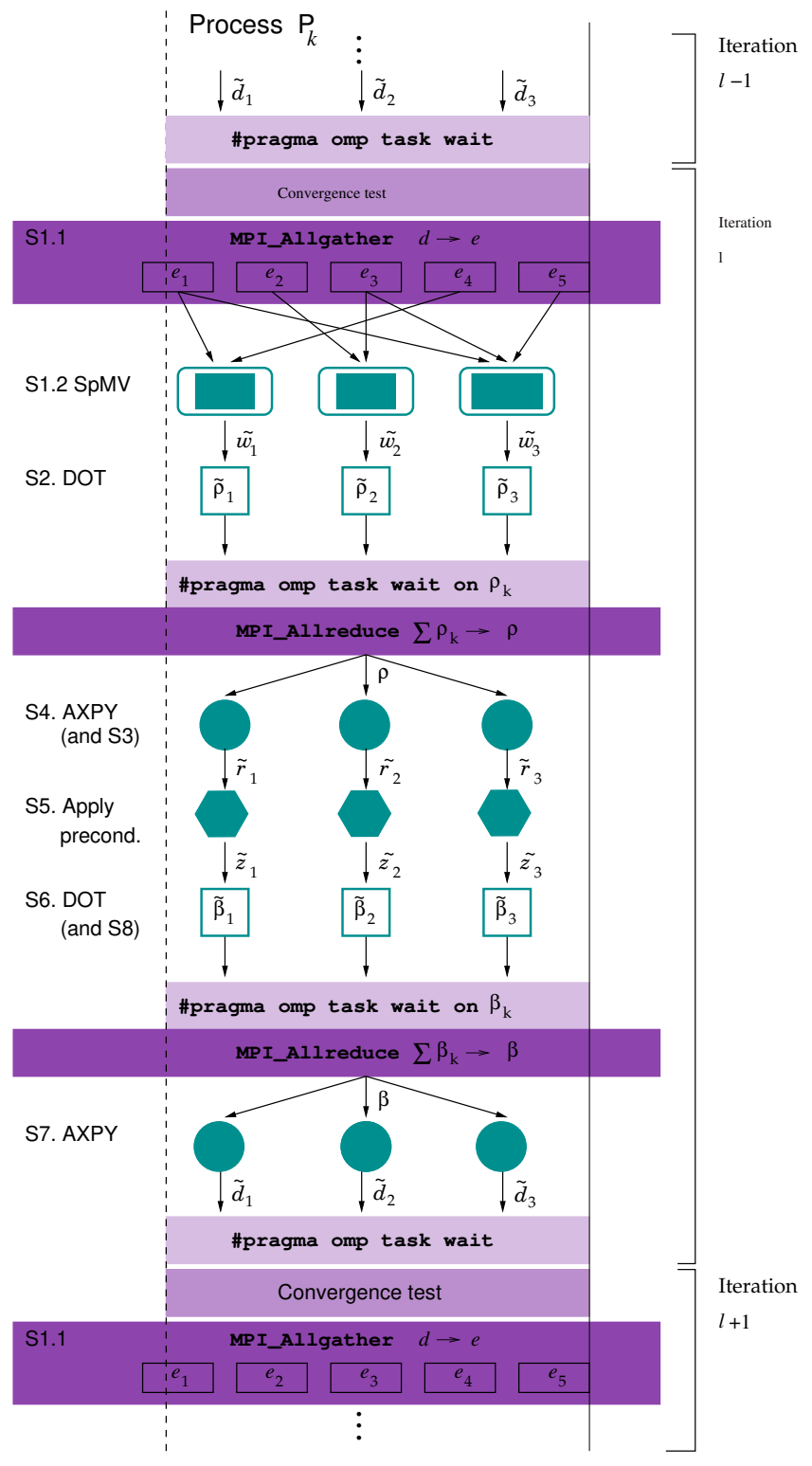

Figure 3. Dependencies between kernels in the PCG solver.

accumulator in case of not enough capacity to store this error.

Listing 1: Process-local DOT product with OpenMP tasks and ExBLAS.

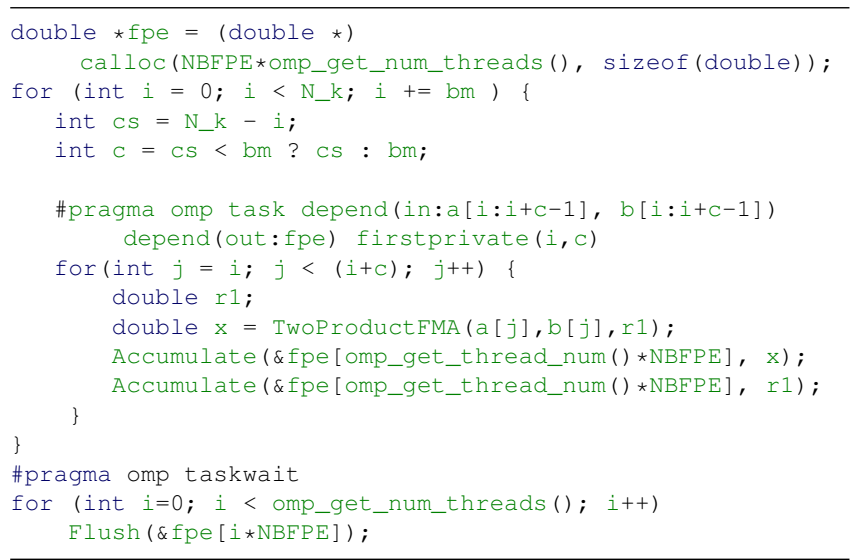

Delivering both correctly rounded and reproducible results, ExBLAS has two major drawbacks Collange et al. 
(2015). The first drawback is related to the required memory storage, which amounts for $n_{t} \times p+a c c_{s}$, where $n_{t}$ is the thread count, $p$ is the size of floating-point expansion, and $a c c_{s}$ is the size of superaccumulator $(2,098$ bits for summation). The second drawback is the number of required operations: For an input vector of size $n$ with dynamic range $d$, the cost of accumulation is

$$
\begin{aligned}
& n \times\left\lceil\frac{d}{52}\right\rceil \times \frac{C_{f p e}}{V L}+n_{t} \times p \times V L \times C_{s a}, \text { when } d<52 p, \\
& n \times\left(p \times \frac{C_{f p e}}{V L}+C_{s a}\right)+n_{t} \times p \times V L \times C_{s a}, \text { otherwise, }
\end{aligned}
$$

where $C_{f p e}=6$ flops, see Algorithm 1, is the cost of the expansion update, $V L$ is the architecture-dependent vector length on SIMD architectures (4 with AVX and 1 on GPUs), and $C_{s a}=16$ flops +2 indirect memory accesses is the cost of the long accumulator update. The right-hand side term is the cost of flushing expansions to long accumulators at the end of the summation and gets negligible as $n$ increases. These two drawbacks can be observed for compute-intensive kernels, leading to large performance overheads Iakymchuk et al. (2016). However, these drawbacks are either hardly visible or relatively small on bandwidth- and memorybound operations such as the DOT product (reduction) and potentially PCG due to the possibility to saturate bandwidth and hide the cost of extra computations and memory needs.

\subsection{FPE-based Strategy}

We introduce a lightweight strategy for reproducibility using the ExBLAS approach as a starting point. The ExBLAS drawbacks served us as a motivation to design an alternative, cheaper strategy for reproducible computations with accuracy (correct rounding) guarantees. Examining the 8 PCG method for moderately conditioned but largely sparse ${ }^{9}$ matrices, like the studied Poisson matrices, we come to the ${ }_{11}$ conclusion that the method can successfully accommodate 12 accurate and reproducible computations, ensuring their robustness, using eight-floating point numbers, meaning the FPE of size 8 (FPE8). In fact, the size of FPEs is tunable and exposed to the end-user. This approach is complemented with the early-exit technique Collange et al. (2015): we stop propagating zero-errors in the FPE. From our experience, the early-exit technique significantly improves performance. According to Hida et al. (2001), FPE8 is capable to represent at least $424=8 \cdot 53$ bits of significant.

Our main motivation for iterative solvers, where next iteration corrects the previous estimate, is to provide a good enough associativity-assuring approach since the properties provided by ExBLAS get demolished by the next computation/iteration. In fact, we are working on developing a concept of weak reproducibility Imamura et al. (2019) - reproducibility under a certain accuracy guarantee, e.g. defined as the input tolerance, that is not necessarily correct rounding. However, we want all computations on a single iteration to be reproducible. Therefore, the FPEs of size 8 with the early-exit technique is generic enough to cover a wide range of problems with various condition numbers and/or dynamic ranges. We also use two different FPEs underneath (one for results and another for errors) that are merged at the end of computations before rounding.

We discuss here the DOT product using OpenMP tasks, while the distributed DOT product is presented in the section below. Listing 2 outlines the FPE-based solution: each MPI process allocates a vector of FPEs for each OpenMP thread and invokes a local routine to conduct DOT products on their local copies of vectors of size $N_{k}$. This local DOT product routine subdivides the process-local DOT product into tasks of size $b m$; each task calls a sequential DOT product. This DOT product as in Listing 1 is composed of the call to twoprod EFT (Algorithm 2) for the exact multiplication of two floating-point numbers; and, then, the accumulation of the output result and the error to the thread local FPEs with the help of Algorithm 4, which relies upon the twosum EFT (Algorithm 1). Later, the FPEs with the result and the error are combined into one by invoking Algorithm 3, which calls Algorithm 4 in a loop over the FPE with errors: Accumulate (fpe, fperr $[i])$. To complete the OpenMP DOT product, we perform the process-local reduction on FPEs by sequentially executing Algorithm 3. Finally, we round the FPE-result to the target precision using the NearSum algorithm Rump et al. (2008b), which is described in Section 4.3.

Listing 2: Process-local DOT product with OpenMP tasks and FPEs.
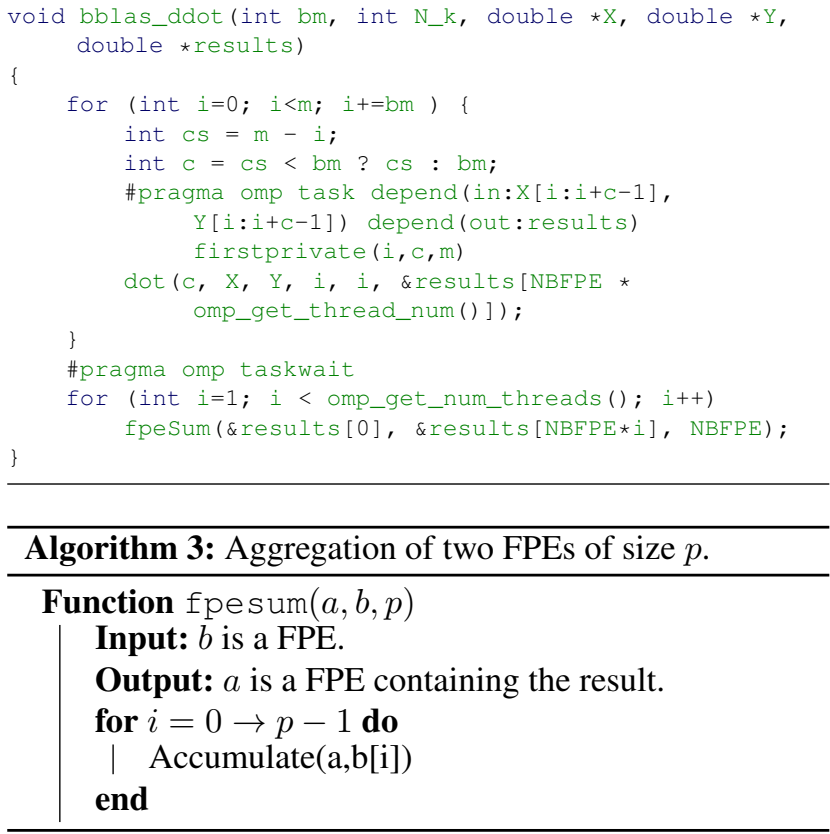

Algorithm 4: Adding a floating-point number $x$ to a floating-point expansion $a$ of size $p$.

Function Accumulate $(a, x)$ Input: $x$ is a floating-point number.

Output: $a$ is a FPE containing the result.

for $i=0 \rightarrow p-1$ do $\mid(a[i], x):=\operatorname{twosum}(a[i], x)$

\subsection{Re-installing Reproducibility of PCG}

We re-assure reproducibility of parallel PCG by first examining potential sources of non-deterministic computations and, in addition, presenting our mitigation strategies for them. Note that we target a hybrid MPI + OpenMP tasks implementation of PCG, where each process conducts computations 
on its own local slices of the matrix as well as the vectors (see Section 3.2 and Figure 2).

DOT products (S2, S6, S8): The main issue of nondeterminism emerges from DOT products and, thus, the parallel reductions such as MPI_Allreduce () that are employed in order to compute the tolerance $\tau$ as well as both $\beta$ and $\rho$. Hence, we 1) exploit the ExBLAS approach to build reproducible and correctly-rounded DOT product; 2) construct DOT product solely based on FPEs; 3) extend the ExBLAS- and FPE-based DOT products to distributed memory in order to make them suitable for the $\mathrm{PCG}^{9}$ algorithm in Figure 2. While Sections 4.1 and 4.2 present implementations of DOT product using OpenMP tasks, i.e. within each MPI process, Listings 3 and 4 provide pseudocodes for our implementation of the distributed DOT product using the ExBLAS and lightweight strategies, respectively. After carrying out process-local DOT products, via either ExBLAS- or FPE-based implementations, we realise the global reduction by splitting them into three stages:

- MPI_Reduce ( ) acting on either long accumulators or FPEs. For the ExBLAS approach, since the long accumulator is an array of long integers, we apply regular reduction. Note that we may need to carry an extra intermediate normalization after the reduction of $2^{K-1}$ long accumulators, where $K=64-52=12$ is the number of carry-safe bits per each digit of long accumulator. For the FPE approach, we may need to renormalize FPEs using the Priest's renormalization method Hida et al. (2001); Priest (1991) and define the MPI operation that is based on the twosum EFT, see Algorithm 3;

- Rounding to double: for long accumulators, we use the ExBLAS-native Round () routine. To guarantee correctly rounded results of the FPE-based computations, we employ the NearSum algorithm from Rump et al. (2008b) for FPEs of size eight or variable size; it may require renormalization before.

- MPI_Bcast ( ) to distribute the result of DOT product to the other processes as only master performs rounding.

Splitting the MPIAllreduce() operation into MPI_Reduce () and MPI_Bcast () provides us full control of the operation and even may lead to better performance as noted in Hunold and Carpen-Amarie (2016).

\section{Listing 3: Reproducible Allreduce with ExBLAS.}

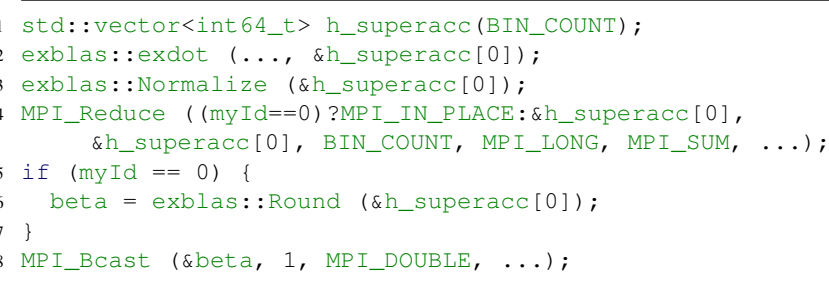

Listing 4: Reproducible Allreduce with FPEs only.

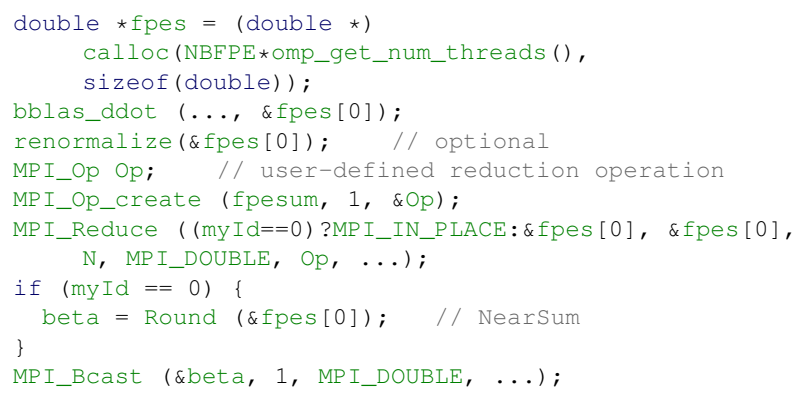

Sparse matrix-vector product (S1): The other reproducibility issue is hidden in the computation of the sparse matrix-vector product. With the current distributed implementation of this operation, each MPI process computes its dedicated part $w_{k}$ of the vector $w$ by multiplying a block of rows $A_{k}$ by the vector $e$. These process-local multiplications are correspondingly divided into tasks, where each task is responsible for a product of a sub-block of rows by the vector. Since the computations are carried locally and sequentially, they are deterministic and, thus, reproducible. However, some parts of the code like $a+=b * c-$ present in the original implementation of PCG - may not always provide with the same result, depending on the compiler optimization strategies.

Our approach to solve this issue is to explicitly instruct compilers to use fma ${ }^{\dagger}$. Note that the underlying architecture should support fma; otherwise, this may lead to the runtime error. This is possible through the std: : fma instruction added to the $\mathrm{C}^{++} 11$ language standard. With this option, we avoid non-determinism in the order of operations, reduce the number of rounding errors from three to two, and, therefore, achieve reproducibility for this type of operations. Consequently, we accomplish reproducibility for the sparse matrix-vector multiplication.

AXPY(-type) vector updates (S3, S4, S7): For this type of operations, we rely upon the sequential MKL implementation of AXPY(-type). Alternatively, we can replace this call to MKL's AXPY(-type) by our implementation using fma to ensure correctly-rounded and, hence, reproducible results. This will not impact performance since the algorithm is strictly memory-bound and this type of kernels are not performance-crucial.

Application of the preconditioner (S5): The application of the Jacobi preconditioner is rather simple: first, the inverse of the diagonals are computed and then the application of the preconditioner only involves element-wise multiplication of two vectors. Thus, this part is both correctly rounded and reproducible.

Reproducibility and accuracy of both approaches: It is evident that the results provided by ExBLAS DOT are both correctly-rounded and reproducible. With the lightweight DOT, we search for the minimal size of FPE such that we still preserve every bit of both the result and the error. For the studied 3D Poisson's equation, the sweet spot is the FPE of size 3, which ensures identical results to ExBLAS and the

$\dagger$ This and the other case of $y=a * x+b * y$ are analyzed in more details in Wiesenberger et al. (2019). 
reference highly accurate solution. However, we aim also to be generic and, hence, we provide the implementation that relies on FPEs of size eight with the early-exit technique. We add a check for the FPE-based implementation for those cases where the condition number and/or the dynamic range are too large and we cannot keep every bit of information. A warning is then raised, offering also a suggestion to switch to the ExBLAS-based implementation. Nonetheless, note that the lightweight implementation is intended for moderately conditioned problems or with moderate dynamic range in order to be accurate, reproducible, but also high performing since the ExBLAS version can be very resource demanding. To sum up, if the information about the problem is know in advance, it is good to explore the FPE-based implementation.

\section{Experimental Results}

\subsection{Setup}

The experiments in this section employed IEEE754 doubleprecision arithmetic and were carried out in two different clusters:

- The MareNostrum4 (MN4) supercomputer at Barcelona Supercomputing Center (BSC): This platform consists of SD530 Compute Racks with an Intel Omni-Path high performance network interconnect. Each node comprises two 24-core Intel Xeon Platinum 8160 processors $(2.10 \mathrm{GHz})$ and 96 Gbytes of DDR4 RAM. The platform runs the SuSE Linux Enterprise Server operating system. The codes in this platform were compiled using GCC v7.2.0, Intel MPI v2018.1, and MKL v2017.4.

- The Tintorrum cluster at Universitat Jaume I: This is a 8-node cluster, where each node is equipped with two 8-core Intel Xeon(R) E5-2630v3 processors (Haswell-EP) (for a total of 128 cores), running at $2.4 \mathrm{GHz}$, with $20 \mathrm{MBytes}$ of L3 on-chip cache (LLC or last level of cache), and with 64 GBytes of DDR3 RAM. The operating system running in the cluster is Linux version 2.6.32-642.4.2.el6.centos.plus.x86_64. The codes were compiled with GCC v5.3.0, OpenMPI v1.10.2, and Intel MKL v2017.1.

For the experimental analysis, we leveraged a sparse s.p.d. coefficient matrix arising from the finite-difference method of a 3D Poisson's equation with 27 stencil points. The fact that the vector involved in the SPMV kernel has to be replicated in all MPI ranks constrains the size of the largest problem that can be solved. Given that the theoretical cost of PCG is $t_{c} \approx 2 n n z+7 n$ floating-point arithmetic operations, where $n n z$ denotes the number of nonzeros of the original matrix and its size $n$, the execution time of the method is usually dominated by that of the SPMV kernel. Therefore, in order to analyze the weak scalability of the method, we maintain the number of non-zero entries per node. For this purpose, we modified the original matrix, transforming it into a band matrix, where the lower and upper bandwidths (bandL and bandU, respectively) depend on the number of nodes employed in the experiment as follows:

$$
\begin{aligned}
\text { band } L=\text { band } U=100 \times \# \text { nodes } \Longrightarrow \\
n n z=(\text { band } L+\text { band } U+1) \times n .
\end{aligned}
$$

With 8 nodes in Tintorrum and 16 in MN4, the bandwidth ranges between 100 and 800 in the first platform, and from 100 to 1,600 in the second one. With this approach we can then maintain the number of rows/columns of the matrix equal to $n=4,000,000$, while increasing its bandwidth and, therefore, the computational workload proportionally to the hardware resources, as required in a weak scaling experiment.

The right-hand side vector $b$ in the iterative solvers was always initialized to the product of $A$ with a vector containing ones only; and the PCG iteration was started with the initial guess $x_{0}=0$. The parameter that controls the convergence of the iterative process was set to $10^{-8}$.

\subsection{Performance Evaluation}

We analyze the performance of two reproducible versions of the PCG algorithm parallelized with MPI: one that relies on the ExBLAS approach, and an alternative variant that is based on floating-point expansions (FPEs) of size eight with the early-exit technique. Hereafter, we will refer to them as Exblas and Opt (or FPE8EE), accordingly. Our experiments evaluate the strong and weak scaling of these reproducible implementations compared against the regular (non-deterministic) version of PCG; all three versions are implemented with MPI + OpenMP tasks.

We next analyze the performance of the three implementations in the aforementioned clusters. On the one hand, in order to assess the strong scalability, we fix the matrix size to $n=16,000,000$ and the size of the upper and lower bandwidth to 100 , as we increase the number of cores. On the other hand, in order to analyze the weak scalability, we proceed as explained earlier, fixing the matrix size to $n=4,000,000$ and increasing the bandwidth from 100 to $100 \times$ mnodes (with mnodes $=16$ in MN4 and mnodes $=8$ in Tintorrum).

Table 2 reports the total execution time (averaged for 5 different executions) of the different MPI + OpenMP tasks PCG solvers on both platforms, varying the number of cores (from 48 to 768 in MN4 and from 16 to 128 in Tintorrum) as we maintain the problem size. We tested different computations of MPI processes per node and OpenMP threads per process: the best performing in MN4 is $8 \mathrm{MPI}$ process with 6 OpenMP threads each, and the optimum combination on Tintorrum is 8 MPI process with 2 OpenMP threads each. The weak scaling experiment offers notable results, as, when executing the algorithms in more than one node (up to 48 cores in MN4 and up to 16 cores on Tintorrum) while increasing proportionally the problem, the execution time is maintained. The executions on one node show a different behavior because the communication is in general faster as it entails no inter-node communication. Notably, these extra (local) operations of both ExBLAS and Opt implementations have a positive effect on scalability on the larger node count due to better ratio of computations to communication compared with the original version. The behaviour of the strong scaling experiment could be expected for a parallel algorithm dealing with a sparse linear algebra operation. This experiment in particular reports an important increase of the overhead when the number of nodes becomes large as the communication cost then dominates the execution time. But, the overhead of the reproducible versions decreases due to the favorable ratio 


\begin{tabular}{|c|c|c|c|c|c|c|}
\hline \multicolumn{7}{|c|}{ Execution time in seconds of the implementations in MN4 } \\
\hline \multirow{2}{*}{$\begin{array}{l}\text { Number } \\
\text { of cores }\end{array}$} & \multicolumn{3}{|c|}{ Weak scaling } & \multicolumn{3}{|c|}{ Strong scaling } \\
\hline & Regular & Exblas & Opt & Regular & Exblas & Opt \\
\hline 48 & $3.5349 \mathrm{E}+00$ & $8.8568 \mathrm{E}+00$ & $7.7153 \mathrm{E}+00$ & $1.3280 \mathrm{E}+01$ & $3.5312 \mathrm{E}+01$ & $2.9730 \mathrm{E}+01$ \\
\hline 96 & $3.1697 \mathrm{E}+00$ & $5.9492 \mathrm{E}+00$ & $5.4720 \mathrm{E}+00$ & $7.6761 \mathrm{E}+00$ & $1.8550 \mathrm{E}+01$ & $1.6142 \mathrm{E}+01$ \\
\hline 192 & $2.9610 \mathrm{E}+00$ & $4.7935 \mathrm{E}+00$ & $4.5801 \mathrm{E}+00$ & $5.1802 \mathrm{E}+00$ & $1.0523 \mathrm{E}+01$ & $9.3799 \mathrm{E}+00$ \\
\hline 384 & $2.8018 \mathrm{E}+00$ & $3.9885 \mathrm{E}+00$ & $3.8810 \mathrm{E}+00$ & $3.9321 \mathrm{E}+00$ & $6.5620 \mathrm{E}+00$ & $6.0571 \mathrm{E}+00$ \\
\hline 768 & $3.5905 \mathrm{E}+00$ & 4.7965E+00 & $4.7348 \mathrm{E}+00$ & $3.6662 \mathrm{E}+00$ & $5.0488 \mathrm{E}+00$ & $4.7846 \mathrm{E}+00$ \\
\hline \multicolumn{7}{|c|}{ Execution time in seconds of the implementations on Tintorrum } \\
\hline \multirow{2}{*}{$\begin{array}{l}\text { Number } \\
\text { of cores }\end{array}$} & \multicolumn{3}{|c|}{ Weak scaling } & \multicolumn{3}{|c|}{ Strong scaling } \\
\hline & Regular & Exblas & Opt & Regular & Exblas & Opt \\
\hline 16 & $8.3203 \mathrm{E}+00$ & $1.4222 \mathrm{E}+01$ & 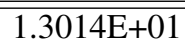 & $3.2747 \mathrm{E}+01$ & $5.7285 \mathrm{E}+01$ & $\overline{5.1238 \mathrm{E}+01}$ \\
\hline 32 & $1.6787 \mathrm{E}+01$ & $2.2833 \mathrm{E}+01$ & $2.1898 \mathrm{E}+01$ & $4.8481 \mathrm{E}+01$ & $7.0335 \mathrm{E}+01$ & $6.8607 \mathrm{E}+01$ \\
\hline 64 & $1.8877 \mathrm{E}+01$ & $2.1114 \mathrm{E}+01$ & $2.0992 \mathrm{E}+01$ & $5.8668 \mathrm{E}+01$ & $7.2928 \mathrm{E}+01$ & $7.1930 \mathrm{E}+01$ \\
\hline 128 & $1.8322 \mathrm{E}+01$ & $2.0331 \mathrm{E}+01$ & $2.0156 \mathrm{E}+01$ & $6.4591 \mathrm{E}+01$ & $6.8174 \mathrm{E}+01$ & $6.7651 \mathrm{E}+01$ \\
\hline
\end{tabular}

Table 2. Timings of different implementations of the Preconditioned Conjugate Gradient method in MN4 and Tintorrum.

between computations and communication. Unfortunately, we cannot evaluate a larger problem to increase the weight of the computational cost, as the problem dimension is constrained by the node memory capacity.

Figure 4 reports the total execution time (averaged for 5 different executions) of the reproducible MPI PCG solvers for the two clusters normalized with respect to the execution time of the regular MPI version, when we vary the number of cores (from 48 to 768 in MN4 and from 16 to 128 in Tintorrum). Specifically, in the two top plots we present the strong scaling evaluation. In these graphs, we can observe that the difference of both versions with respect to the regular one is higher on a small number of cores, and it decreases with the core count. We observe that the overhead of both the Exblas and Opt implementations compared with the regular version is smooth and decreasing: from $2.66 \mathrm{x}$ and $2.24 \mathrm{x}$ on the single node to $37.7 \%$ and $30.5 \%$ on 16 nodes on MN4 for Exblas and Opt, respectively; and, on Tintorrum from $74.9 \%$ and $56.5 \%$ on the single node to $5.6 \%$ and $4.7 \%$ on 8 nodes for Exblas and $O p t$, accordingly. Moreover, the overhead between Exblas and Opt versions decreases, e.g. from $18 \%$ to $6 \%$ in MN4, on the large core count: this is due to very similar implementations of both since Exblas underneath relies upon FPE8EE for the OpenMP DOT products. Note that such difference is much larger for the pure MPI implementation Iakymchuk et al. (2019a).

The two bottom graphs in Figure 4 expose the weak scaling evaluation, where we set the number of nonzeros of the sparse matrix to be roughly proportional to the number of cores, increasing the size of the band of the matrix, as discussed in Section 5.1. These results show that both versions offer similar performance to the baseline on the large number of cores. For instance, the overheads are $33.70 \%$ and $31.75 \%$ for the Exblas and $O p t$ implementations in MN4, respectively, and only $11 \%$ and $10 \%$ for Exblas and Opt on Tintorrum, accordingly. As in the strong scaling analysis, the Opt version outperforms the Exblas implementation. If we compare the results in both clusters, we can observe that they are more stable in Tintorrum because the number of cores per node is smaller in this platform than in MN4.

\subsection{Accuracy and Reproducibility Evaluation}

In addition to the performance results, we report also the results of the accuracy and reproducibility evaluation. For that, we develop a generator of ill-conditioned matrices. This generator scales the first row and the first column of the matrix so that the DOT product determines the condition number of the matrix. Additionally, we derive a sequential version of the code that relies on the GNU Multiple Precision Floating-Point Reliably (MPFR) library Fousse et al. (2007) - a C library for multiple (arbitrary) precision floating-point computations on CPUs - as a highly accurate reference implementation. This implementation uses 2,048 bits of accuracy for computing the DOT product (192 bits for internal product of two floating-point numbers) and performs correct rounding of the computed result to double precision.

Table 3 reports the intermediate and final residual on each iteration of the PCG solver for the matrix with the number of rows/columns equal to $n=4,019,679\left(159^{3}\right)$, the bandwidth of size 200 , and the condition number of $10^{12}$. The results are exposed with all digits in hexadecimal. For this test, the tolerance was set to $10^{-8}$ and it took 47 iterations for all four implementations to converge under this accuracy requirement. We used one node of MN4 with 48 processes each pinned to one core. We present only few iterations, but the difference is present in all iterations. The ExBLAS and Opt implementations deliver both accurate and reproducible results that are identical with the MPFR library. Note that these results are identical to the ones from the pure MPI implementations in Iakymchuk et al. (2019a) and only the results of the original code differ. The original code shows the difference from one digit on the initial iteration and up to five digits on the 45 th iteration on 48 cores ( 8 MPI processes with 6 OpenMP threads per each). We also add the results of the original code on one core/process to highlight the reproducibility issue. To show these results, we merge the two columns of the ExBLAS and Opt results as they are identical.

We assume that this discrepancy in accuracy and reproducibility becomes larger at scale (more nodes) due to the stronger impact of the topology and reduction trees. 
Strong Scalability on MareNostrum4

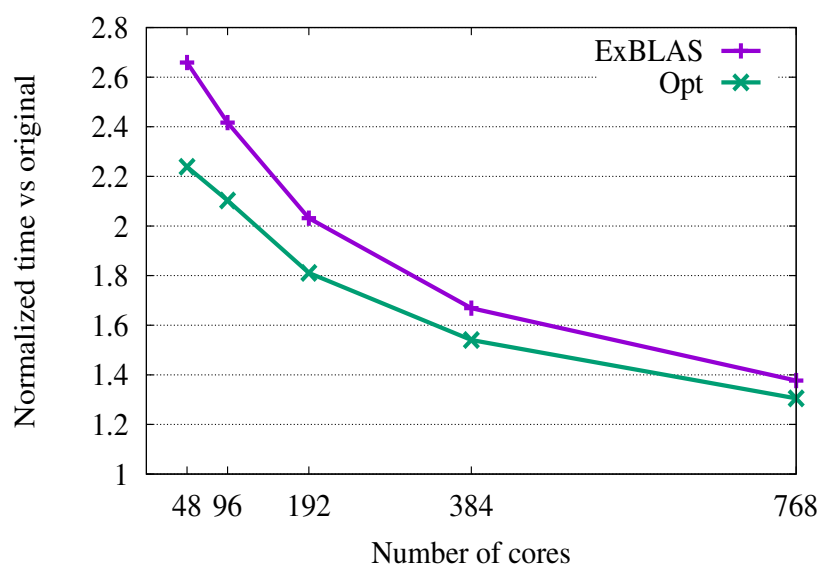

Weak Scalability on MareNostrum4

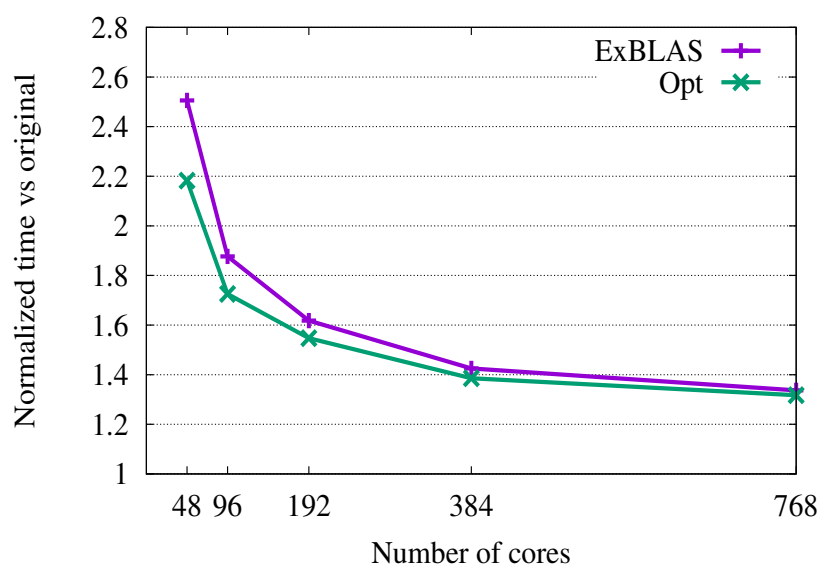

Strong Scalability on Tintorrum

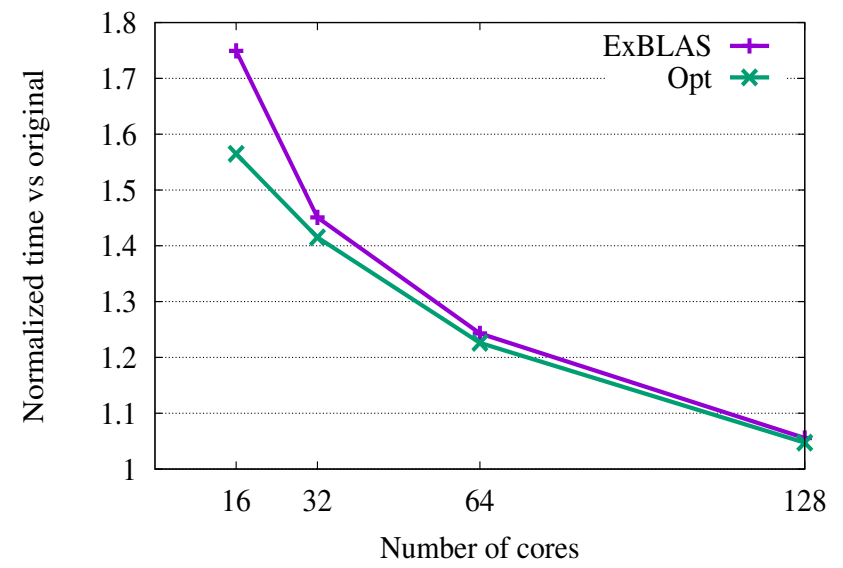

Weak Scalability on Tintorrum

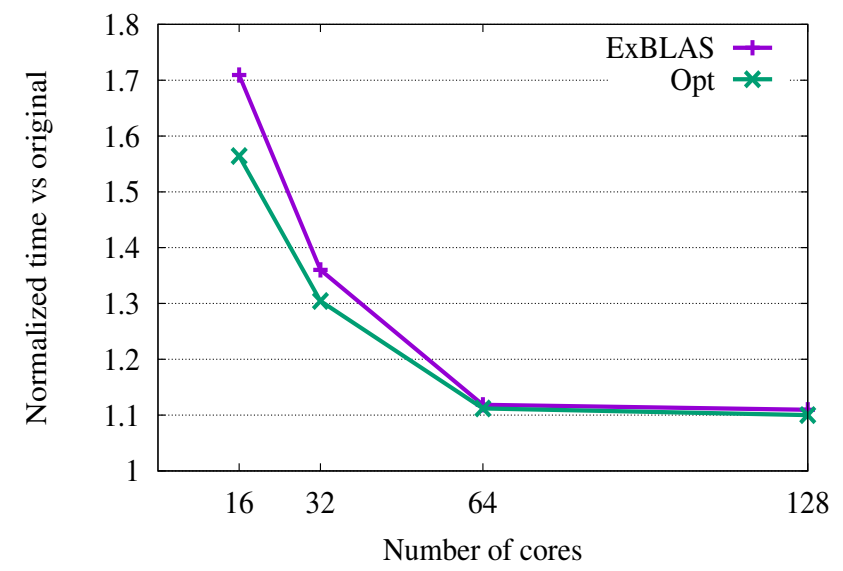

Figure 4. Analysis of the strong (top) and weak (bottom) scalability of the two reproducible versions of the MPI + OpenMP tasks PCG; the time is normalized with respect to the regular non-deterministic MPI version.

\begin{tabular}{|c|l|l|l|l|}
\hline \multirow{2}{*}{ Iteration } & \multicolumn{4}{|c|}{ Residual } \\
\cline { 2 - 5 } & MPFR & Original 1 core & Original 48 cores & Exblas \& FPE8EE \\
\hline \hline 0 & 0x1.19f179eb7f032p+49 & 0x1.19f179eb7f033p+49 & 0x1.19f179eb7f033p+49 & 0x1.19f179eb7f032p+49 \\
2 & 0x1.f86089ece9f75p+38 & 0x1.f86089ece5bd4p+38 & 0x1.f86089eceaf76p+38 & 0x1.f86089ece9f75p+38 \\
9 & 0x1.fc59a29d329ffp+28 & 0x1.fc59a29d3599ap+28 & 0x1.fc59a29d32d1bp+28 & 0x1.fc59a29d329ffp+28 \\
10 & 0x1.74f5ccc211471p+22 & 0x1.74f5ccc1d03cbp+22 & 0x1.74f5ccc201246p+22 & 0x1.74f5ccc211471p+22 \\
$\ldots$ & $\ldots$ & $\ldots$ & $\ldots$ & $\ldots$ \\
40 & 0x1.7031058eb2e3ep-19 & 0x1.7031058dd6bcfp-19 & 0x1.7031058eaf4c2p-19 & 0x1.7031058eb2e3ep-19 \\
42 & 0x1.4828f76bd68afp-23 & 0x1.4828f76d1aa3p-23 & 0x1.4828f76bda71ap-23 & 0x1.4828f76bd68afp-23 \\
45 & 0x1.8646260a70678p-26 & 0x1.8646260a2dae8p-26 & 0x1.8646260a6da06p-26 & 0x1.8646260a70678p-26 \\
47 & 0x1.13fa97e2419c7p-33 & 0x1.13fa97e1e76bfp-33 & 0x1.13fa97e240f7cp-33 & 0x1.13fa97e2419c7p-33 \\
\hline
\end{tabular}

Table 3. Accuracy and reproducibility comparison on the intermediate and final residual against MPFR for a matrix with the condition number of $10^{12}$. The matrix is generated following the procedure from Section 5.1 with $n=4,019,679\left(159^{3}\right)$ and the bandwidth of size 200 .

\subsection{Evaluation using the SuiteSparse matrix collection}

We conduct a set of tests using real cases from the SuiteSparse matrix collection. We select matrices with various condition numbers starting from $1.49 e+04$ up to $2.22 e+18^{\ddagger}$, with as many as one million nonzero elements. Table 4 presents our experimental results on a single node using eight MPI processes and six OpenMP threads per process on the MareNostrum4 cluster. For each test matrix, we report the number of iterations required to reach the tolerance of $10^{-8}$ for residual, the direct error computed as in
Section 3.5.1 Golub and Loan (2013), and the total execution time. We have selected the direct error instead of residual since it is known that smaller residual does not imply higher accuracy, meaning solutions with smaller residual might be less accurate. This is confirmed for the first three matrices for which the residual suffices the tolerance but the direct error is still large. The direct error as well as the number of iterations

\footnotetext{
$\ddagger$ In practice, selecting coefficient matrices for the linear systems for which $\operatorname{cond}(A) \leq 1 e+12$ would have been more realistic due to the limits of the double precision arithmetic.
} 
are identical for both ExBLAS and Opt variants, hence we merge these columns. Our reproducible variants require a smaller number of iterations than the original version for the msc01050, olafu, and bcsstk28 matrices. For instance, for the olafu matrix $(1,015,156$ nonzero elements and condition number $7.61 e+11$ ), both ExBLAS and Opt variants require 42,342 iterations, while the original version needs 43,046 iterations. For a few cases, our reproducible variants may perform slightly more iterations than the non-reproducible variants due to the differences in the accumulation of rounding errors arising form distinct optimizations in the codes. The overhead of our reproducible variants can be as low as $32.5 \%$ for the 494_bus matrix but can reach $3.46 \times$ for the sts4098 matrix. This overhead is expected and is inline with the pattern from Figure 4, where the largest overhead is observed on a single node. Moreover, we also run tests using eight MPI processes and two OpenMP threads per process the $O p t$ and ExBLAS results are again identical in terms of the number of iterations, residuals, and direct errors.

Furthermore, we conduct similar experiments on the Tintorrum cluster. Table 5 presents the results of these experiments. There, we also use one node but four MPI processes and four OpenMP threads per process. These results show a similar trend to that of MareNostrum4: smaller number of iterations of the ExBLAS and $O p t$ versions for plat1919, olafu, gyro_k, bcsstk28, and msc04515 matrices; the overhead of reproducible versions as small as $88.1 \%$ for the 494_bus matrix but may also grow up to few times. Notably, both ExBLAS and Opt versions deliver identical results, excluding timings, on the MareNostrum4 and Tintorrum clusters.

In addition, we conduct experiments using the pure MPI versions of the Reproducible Preconditioned Conjugate Gradient Iakymchuk et al. (2019a) on the MareNostrum4 and Tintorrum clusters, see Tables 6 and 7. We observe that the number of iterations, residuals, direct errors, the final error, and vector-solutions are identical to those produced by the MPI+OpenMP tasks versions. Hence, our reproducible strategies ensure cross-cluster reproducibility of PCG implemented with pure MPI as well as the hybrid MPI + OpenMP tasks models.

\section{Related Work}

To enhance reproducibility, Intel proposed the "Conditional Numerical Reproducibility" (CNR) option in its Math Kernel Library (MKL). Although CNR guarantees reproducibility, it does not ensure correct rounding, meaning the accuracy is arguable. Additionally, the cost of obtaining reproducible results with CNR is high. For instance, for large arrays the MKL's summation with CNR was almost $2 x$ slower than the regular MKL's summation on the Mesu cluster hosted at the Sorbonne University Collange et al. (2015).

Demmel and Nguyen implemented a family of algorithms - that originate from the works by Rump, Ogita, and Oishi Rump et al. (2010, 2008b,a) - for reproducible summation in floating-point arithmetic Demmel and Nguyen (2013, 2015). These algorithms always return the same answer. They first compute an absolute bound of the sum and then round all numbers to a fraction of this bound. In consequence, the addition of the rounded quantities is exact, however the computed sum using their implementations with two or three bins is not correctly rounded. Their results yielded roughly $20 \%$ overhead on 1024 processors (CPUs only) compared to the Intel MKL dasum (), but it shows 3.4 times slowdown on 32 processors (one node). Ahrens, Nguyen, and Demmel extended their concept to few other reproducible BLAS routines, distributed as the ReproBLAS library ${ }^{\S}$, but only with parallel reproducible reduction. Furthermore, the ReproBLAS effort was extended to reproducible tall-skinny QR Nguyen and Demmel (2015).

The other approach to ensure reproducibility is called ExBLAS, which is initially proposed by Collange, Defour, Graillat, and Iakymchuk in Collange et al. (2015). ExBLAS is based on combining long accumulators and floating-point expansions in conjuction with error-free transformations. This approach is presented in Section 2.1. Collange et al. showed Collange et al. (2015) that their algorithms for reproducible and accurate summation have $8 \%$ overhead on 512 cores ( 32 nodes) and less than $2 \%$ overhead on 16 cores (one node). While ExSUM covers wide range of architectures as well as distributed-memory clusters, the other routines primarily target GPUs. Exploiting the modular and hierarchical structure of linear algebra algorithms, the ExBLAS approach was applied to construct reproducible LU factorizations with partial pivoting Iakymchuk et al. (2019b).

Recently, Mukunoki and Ogita presented their approach to implement reproducible BLAS, called OzBLAS Mukunoki et al. (2020), with tunable accuracy. This approach is different from both ReproBLAS and ExBLAS as it does not require to implement every BLAS routine from scratch but relies on high-performance (vendor) implementations. Hence, OzBLAS implements the Ozaki scheme Ozaki et al. (2012) that follows the fork-join approach: the matrix and vector are split (each element is sliced) into sub-matrices and sub-vectors for secure products without overflows; then, the high-performance BLAS is called on each of these splits; finally, the results are merged back using, for instance, the NearSum algorithm. Currently, the OzBLAS library includes dot product, matrix-vector product (gemv), and matrixmatrix multiplication (gemm). These algorithmic variants and their implementations on GPUs and CPUs (only dot) reassure reproducibility of the BLAS kernels as well as make the accuracy tunable up-to correctly rounded results.

\section{Conclusions and Future Work}

In this work, we addressed the reproducibility of iterative solvers for sparse linear systems using a representative instance of the Preconditioned Conjugate Gradient method. We first analyzed the hybrid MPI + OpenMP tasks implementation of the PCG method and identified two major sources of non-deterministic behavior, namely the DOT product and compiler optimizations. The latter may change the order of operations or replace some of them in favor of the fused multiply-add (fma) operation. For reproducible and double-layered distributed DOT product, we leveraged the ExBLAS-approach as well as proposed an alternative lightweight variant based solely on FPEs. Both strategies split the MPI_Allreduce routine into the combination of

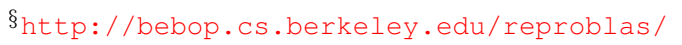




\begin{tabular}{|c|c|c|c|c|c|c|c|c|c|}
\hline \multirow[t]{2}{*}{ Matrix } & \multirow[t]{2}{*}{ Nonzeros } & \multirow[t]{2}{*}{$\operatorname{cond}(A)$} & \multicolumn{2}{|c|}{ Iterations } & \multicolumn{2}{|c|}{ Direct error } & \multicolumn{3}{|c|}{ Time [secs] } \\
\hline & & & Orig & Opt\&ExBLAS & Orig & $O p t \& E x B L A S$ & Orig & Opt & Exblas \\
\hline plat1919 & 32,399 & $2.22 \mathrm{e}+18$ & 28200 & 28347 & 0x1.d1fd2948ac992p+4 & 0x1.d1fd1980ddc3dp+4 & $2.89 \mathrm{e}+00$ & $5.79 \mathrm{e}+00$ & $6.74 \mathrm{e}+00$ \\
\hline $\mathrm{msc} 01050$ & 26,198 & $9.00 \mathrm{e}+15$ & 1459 & 1441 & 0x1.fe62a1a8f70acp-7 & 0x1.c06963286be9ap-7 & $1.35 \mathrm{e}-01$ & $2.27 \mathrm{e}-01$ & $2.48 \mathrm{e}-01$ \\
\hline mhd $4800 \mathrm{~b}$ & 27.520 & $1.03 \mathrm{e}+14$ & 32 & 32 & $0 x 1.171 \mathrm{f} 2 \mathrm{~d} 2 \mathrm{a} 7 \mathrm{c} 15 \mathrm{dp}-7$ & 0x1.171f2cf90e554p-7 & $3.76 \mathrm{e}-03$ & $1.10 \mathrm{e}-02$ & $1.30 \mathrm{e}-02$ \\
\hline olafu & $1,015,156$ & $7.61 \mathrm{e}+11$ & 43046 & 42342 & 0x1.4683bc1ddab86p-28 & 0x1.3c603001a3c8fp-28 & $3.54 \mathrm{e}+01$ & $6.84 \mathrm{e}+01$ & $7.60 \mathrm{e}+01$ \\
\hline gyro_k & $1,021,159$ & $1.10 \mathrm{e}+09$ & 16064 & 16075 & $0 \times 1.7 f 300 f 81 \mathrm{c} 65 \mathrm{c} 7 \mathrm{p}-26$ & 0x1.7ef8863fde778p-26 & $1.41 \mathrm{e}+01$ & $2.78 \mathrm{e}+01$ & $3.10 \mathrm{e}+01$ \\
\hline bcsstk28 & 219,024 & $6.28 \mathrm{e}+09$ & 5592 & 5483 & 0x1.dd4e900472e $3 \mathrm{dp}-31$ & 0x1.ba21beeb93c43p-31 & $1.25 \mathrm{e}+00$ & $2.51 \mathrm{e}+00$ & $2.85 \mathrm{e}+00$ \\
\hline bcsstk13 & 83,883 & $5.64 \mathrm{e}+08$ & 2571 & 2571 & 0x1.03a9e5339d79fp-34 & 0x1.5e81334a6997bp-34 & $3.66 \mathrm{e}-01$ & $6.47 \mathrm{e}-01$ & $7.37 \mathrm{e}-01$ \\
\hline sts4098 & 72,356 & $3.56 \mathrm{e}+07$ & 666 & 668 & 0x1.ad954060aba53p-38 & 0x1.5a2eda56201fbp-38 & $8.87 \mathrm{e}-02$ & $2.28 \mathrm{e}-01$ & $2.61 \mathrm{e}-01$ \\
\hline 494_bus & 1,666 & $3.89 \mathrm{e}+06$ & 410 & 410 & $0 x 1.22$ befca9188e $3 p-35$ & 0x1.b83293969f70bp-36 & $3.29 \mathrm{e}-02$ & $4.36 \mathrm{e}-02$ & $5.05 \mathrm{e}-02$ \\
\hline $\mathrm{msc} 04515$ & 97,707 & $4.78 \mathrm{e}+05$ & 4883 & 4885 & 0x1.41d64ef6a77bfp-32 & 0x1.4b0c4d82346dap-32 & $6.85 \mathrm{e}-01$ & $1.80 \mathrm{e}+00$ & $2.07 \mathrm{e}+00$ \\
\hline bcsstk27 & 56,126 & $1.49 \mathrm{e}+04$ & 331 & 331 & $0 \times 1.3 f 45 a 221626 f d p-40$ & $0 \times 1.2 \mathrm{~b} 65 \mathrm{be} 5099 \mathrm{a} 69 \mathrm{p}-40$ & $3.66 \mathrm{e}-02$ & $5.94 \mathrm{e}-02$ & $6.60 \mathrm{e}-02$ \\
\hline
\end{tabular}

Table 4. Evaluation of different $M P I+$ OpenMP tasks implementations of the PCG method using test matrices from the

SuiteSparse matrix collection on one MareNostrum4's node with eight MPI processes and six OpenMP threads per process.

\begin{tabular}{|c|c|c|c|c|c|c|c|c|c|}
\hline \multirow[t]{2}{*}{ Matrix } & \multirow[t]{2}{*}{ Nonzeros } & \multirow[t]{2}{*}{$\operatorname{cond}(A)$} & \multicolumn{2}{|c|}{ Iterations } & \multicolumn{2}{|c|}{ Direct error } & \multicolumn{3}{|c|}{ Time [secs] } \\
\hline & & & Orig & Opt\&ExBLAS & Orig & Opt\&ExBLAS & Orig & Opt & Exblas \\
\hline plat1919 & 32,399 & $2.22 \mathrm{e}+18$ & 29133 & 28347 & 0x1.d1fccb48b0708p+4 & 0x1.d1fd1980ddc3dp+4 & $2.21 \mathrm{e}+00$ & $6.07 \mathrm{e}+00$ & $7.65 \mathrm{e}+00$ \\
\hline $\mathrm{msc} 01050$ & 26,198 & $9.00 \mathrm{e}+15$ & 1441 & 1441 & $0 \times 1.20$ ef 1 ec5aba0fp-6 & 0x1.c06963286be9ap-7 & $1.07 \mathrm{e}-01$ & $2.26 \mathrm{e}-01$ & $2.74 \mathrm{e}-01$ \\
\hline mhd $4800 \mathrm{~b}$ & 27,520 & $1.03 e+14$ & 32 & 32 & 0x1.171f $2 \mathrm{~d} 405896 \mathrm{bp}-7$ & $0 \times 1.171 \mathrm{f} 2 \mathrm{cf} 90 \mathrm{e} 554 \mathrm{p}-7$ & $4.35 \mathrm{e}-02$ & $1.06 \mathrm{e}-01$ & $8.98 \mathrm{e}-02$ \\
\hline olafu & $1,015,156$ & $7.61 e+11$ & 44309 & 42342 & 0x1.580f68bcf7c59p-28 & $0 \times 1.3 \mathrm{c} 603001 \mathrm{a} 3 \mathrm{c} 8 \mathrm{fp}-28$ & $5.80 \mathrm{e}+01$ & $1.10 \mathrm{e}+02$ & $1.28 \mathrm{e}+02$ \\
\hline gyro_k & $1,021,159$ & $1.10 \mathrm{e}+09$ & 16623 & 16075 & $0 \times 1.70 \mathrm{c} 410 \mathrm{f} 76 \mathrm{c} 1 \mathrm{f} 3 \mathrm{p}-26$ & 0x1.7ef8863fde778p-26 & $2.12 \mathrm{e}+01$ & $4.24 \mathrm{e}+01$ & $4.98 \mathrm{e}+01$ \\
\hline bcsstk 28 & 219,024 & $6.28 \mathrm{e}+09$ & 5485 & 5483 & 0x1.b33c0d0a65819p-31 & 0x1.ba21beeb93c43p-31 & $1.59 \mathrm{e}+00$ & $3.50 \mathrm{e}+00$ & $4.17 \mathrm{e}+00$ \\
\hline bcsstk13 & 83,883 & $5.64 \mathrm{e}+08$ & 2554 & 2571 & 0x1.1f45539d3ad76p-34 & 0x1.5e81334a6997bp-34 & $3.87 \mathrm{e}-01$ & $8.04 \mathrm{e}-01$ & $9.58 \mathrm{e}-01$ \\
\hline sts 4098 & 72,356 & $3.56 \mathrm{e}+07$ & 666 & 668 & $0 x 1.75 \mathrm{~b} 26 \mathrm{e} 5 \mathrm{cc} 575 \mathrm{ep}-38$ & 0x1.5a2eda56201fbp-38 & $1.02 \mathrm{e}-01$ & $3.00 \mathrm{e}-01$ & $3.69 \mathrm{e}-01$ \\
\hline 494_bus & 1,666 & $3.89 \mathrm{e}+06$ & 409 & 410 & 0x1.8a70c6145af0bp-33 & 0x1.b83293969f70bp-36 & $1.76 \mathrm{e}-02$ & $3.31 \mathrm{e}-02$ & $4.14 \mathrm{e}-02$ \\
\hline $\operatorname{msc} 04515$ & 97,707 & $4.78 \mathrm{e}+05$ & 5138 & 4885 & $0 x 1.318 \mathrm{ee} 7 \mathrm{cc} 28729 \mathrm{p}-32$ & 0x1.4b0c4d82346dap-32 & $8.34 \mathrm{e}-01$ & $2.43 e+00$ & $2.98 \mathrm{e}+00$ \\
\hline bcsstk 27 & 56,126 & $1.49 \mathrm{e}+04$ & 331 & 331 & $0 \times 1.287 \mathrm{~d} 86 \mathrm{ae} 5 \mathrm{~b} 307 \mathrm{p}-40$ & 0x1.2b65be $5099 \mathrm{a} 69 \mathrm{p}-40$ & $3.34 \mathrm{e}-02$ & $6.53 \mathrm{e}-02$ & $7.81 \mathrm{e}-02$ \\
\hline
\end{tabular}

Table 5. Evaluation of different $\mathrm{MPI}+$ OpenMP tasks implementations of the PCG method using test matrices from the SuiteSparse matrix collection on one Tintorrum's node with four MPI processes and four OpenMP threads per process.

\begin{tabular}{|c|c|c|c|c|c|c|c|c|c|}
\hline \multirow[t]{2}{*}{ Matrix } & \multirow[t]{2}{*}{ Nonzeros } & \multirow[t]{2}{*}{$\operatorname{cond}(A)$} & \multicolumn{2}{|c|}{ Iterations } & \multicolumn{2}{|c|}{ Direct error } & \multicolumn{3}{|c|}{ Time [secs] } \\
\hline & & & Orig & $O p t \& E x B L A S$ & Orig & $O p t \& E x B L A S$ & Orig & $O p t$ & Exblas \\
\hline plat1919 & 32,399 & $2.22 \mathrm{e}+18$ & 28404 & 28347 & 0x1.d1fd13459efb2p+4 & 0x1.d1fd1980ddc3dp+4 & $2.09 \mathrm{e}+00$ & $7.25 \mathrm{e}+00$ & $1.15 \mathrm{e}+01$ \\
\hline $\operatorname{msc} 01050$ & 26,198 & $9.00 \mathrm{e}+15$ & 1449 & 1441 & 0x1.1e960e3dd96adp-6 & 0x1.c06963286be9ap-7 & $1.18 \mathrm{e}-01$ & $3.51 \mathrm{e}-01$ & $4.95 \mathrm{e}-01$ \\
\hline mhd $4800 \mathrm{~b}$ & 27,520 & $1.03 \mathrm{e}+14$ & 32 & 32 & $0 \times 1.171 \mathrm{f} 2 \mathrm{~d} 513 \mathrm{bf} 1 \mathrm{fp}-7$ & $0 \times 1.171 \mathrm{f} 2 \mathrm{cf} 90 \mathrm{e} 554 \mathrm{p}-7$ & $5.17 \mathrm{e}-03$ & $9.00 \mathrm{e}-03$ & $2.16 \mathrm{e}-02$ \\
\hline olafu & $1,015,156$ & $7.61 \mathrm{e}+11$ & 44872 & 42342 & 0x1.284b2460347acp-28 & $0 \times 1.3 \mathrm{c} 603001 \mathrm{a} 3 \mathrm{c} 8 \mathrm{fp}-28$ & $1.38 \mathrm{e}+01$ & $2.22 \mathrm{e}+01$ & $7.77 \mathrm{e}+01$ \\
\hline gyro_k & $1,021,159$ & $1.10 \mathrm{e}+09$ & 16577 & 16075 & $0 \times 1.76957 \mathrm{c} 0$ ecf952p-26 & $0 \times 1.7$ ef 8863 fde $778 \mathrm{p}-26$ & $5.29 e+00$ & $8.71 e+00$ & $3.14 \mathrm{e}+01$ \\
\hline bcsstk 28 & 219,024 & $6.28 \mathrm{e}+09$ & 5736 & 5483 & 0x1.e9c64a28a93dfp-31 & 0x1.ba21beeb93c43p-31 & $8.43 \mathrm{e}-01$ & $1.70 \mathrm{e}+00$ & $3.69 \mathrm{e}+00$ \\
\hline bcsstk13 & 83,883 & $5.64 \mathrm{e}+08$ & 2571 & 2571 & 0x1.03a9e5339d79fp-34 & 0x1.5e81334a6997bp-34 & $2.32 \mathrm{e}-01$ & $5.07 \mathrm{e}-01$ & $3.01 \mathrm{e}+00$ \\
\hline sts4098 & 72,356 & $3.56 \mathrm{e}+07$ & 666 & 668 & 0x1.ad954060aba53p-38 & 0x1.5a2eda56201fbp-38 & $6.44 \mathrm{e}-02$ & $1.85 \mathrm{e}-01$ & $1.47 \mathrm{e}+00$ \\
\hline 494_bus & 1,666 & $3.89 \mathrm{e}+06$ & 410 & 410 & $0 x 1.98 \mathrm{~d} 21 \mathrm{a} 409 \mathrm{a} 23 \mathrm{cp}-36$ & 0x1.b83293969f70bp-36 & $4.47 \mathrm{e}-02$ & $9.29 \mathrm{e}-02$ & $1.20 \mathrm{e}-01$ \\
\hline $\mathrm{msc} 04515$ & 97,707 & $4.78 \mathrm{e}+05$ & 4883 & 4885 & 0x1.41d64ef6a77bfp-32 & $0 \times 1.4 \mathrm{~b} 0 \mathrm{c} 4 \mathrm{~d} 82346 \mathrm{dap}-32$ & $4.89 \mathrm{e}-01$ & $1.47 \mathrm{e}+00$ & $1.18 \mathrm{e}+01$ \\
\hline bcsstk 27 & 56,126 & $1.49 \mathrm{e}+04$ & 331 & 331 & $0 \times 1.3 f 45 a 221626 f d p-40$ & 0x1.2b65be $5099 \mathrm{a} 69 \mathrm{p}-40$ & $2.02 \mathrm{e}-02$ & $4.50 \mathrm{e}-02$ & $1.22 \mathrm{e}-01$ \\
\hline
\end{tabular}

Table 6. Evaluation of different pure MPI implementations lakymchuk et al. (2019a) of the PCG method using test matrices from the SuiteSparse matrix collection on one MareNostrum4's node with 48 MPI processes.

\begin{tabular}{|c|c|c|c|c|c|c|c|c|c|}
\hline \multirow[t]{2}{*}{ Matrix } & \multirow[t]{2}{*}{ Nonzeros } & \multirow[t]{2}{*}{$\operatorname{cond}(A)$} & \multicolumn{2}{|c|}{ Iterations } & \multicolumn{2}{|c|}{ Direct error } & \multicolumn{3}{|c|}{ Time [secs] } \\
\hline & & & Orig & $O p t \& E x B L A S$ & Orig & $O p t \& E x B L A S$ & Orig & Opt & Exblas \\
\hline plat1919 & 32,399 & $2.22 \mathrm{e}+18$ & 28225 & 28347 & 0x1.d1fd2b1f22f89p+4 & 0x1.d1fd1980ddc3dp+4 & $1.05 \mathrm{e}+00$ & $2.27 \mathrm{e}+00$ & $1.21 \mathrm{e}+01$ \\
\hline $\mathrm{msc} 01050$ & 26,198 & $9.00 \mathrm{e}+15$ & 1440 & 1441 & $0 x 1.91 \mathrm{eb} 8 \mathrm{c} 4 \mathrm{cf} 549 \mathrm{ep}-7$ & 0x1.c06963286be9ap-7 & $5.85 \mathrm{e}-02$ & $1.01 \mathrm{e}-01$ & $4.14 \mathrm{e}-01$ \\
\hline mhd $4800 \mathrm{~b}$ & 27,520 & $1.03 \mathrm{e}+14$ & 32 & 32 & 0x1.171f2d071bbecp-7 & $0 \times 1.171 \mathrm{f} 2 \mathrm{cf} 90 \mathrm{e} 554 \mathrm{p}-7$ & $2.05 \mathrm{e}-03$ & $4.82 \mathrm{e}-03$ & $3.28 \mathrm{e}-02$ \\
\hline olafu & $1,015,156$ & $7.61 \mathrm{e}+11$ & 44840 & 42342 & 0x1.73ee2c0ee4f91p-28 & $0 \times 1.3 \mathrm{c} 603001 \mathrm{a} 3 \mathrm{c} 8 \mathrm{fp}-28$ & $2.08 \mathrm{e}+01$ & $3.39 \mathrm{e}+01$ & $1.55 \mathrm{e}+02$ \\
\hline gyro_k & $1,021,159$ & $1.10 \mathrm{e}+09$ & 16518 & 16075 & $0 \times 1.7 \mathrm{c} 04191 \mathrm{~d} 8 \mathrm{~b} 8 \mathrm{~d} 9 \mathrm{p}-26$ & 0x1.7ef8863fde778p-26 & $8.31 \mathrm{e}+00$ & $1.38 \mathrm{e}+01$ & $6.28 \mathrm{e}+01$ \\
\hline bcsstk 28 & 219,024 & $6.28 \mathrm{e}+09$ & 5640 & 5483 & 0x1.d8e49bef6a637p-31 & 0x1.ba21beeb93c43p-31 & $5.58 \mathrm{e}-01$ & $1.06 \mathrm{e}+00$ & $5.46 \mathrm{e}+00$ \\
\hline bcsstk13 & 83,883 & $5.64 \mathrm{e}+08$ & 2337 & 2571 & 0x1.040b38e017aeep-34 & $0 \times 1.5 \mathrm{e} 81334 \mathrm{a} 6997 \mathrm{bp}-34$ & $1.36 \mathrm{e}-01$ & $2.68 \mathrm{e}-01$ & $1.23 \mathrm{e}+00$ \\
\hline sts4098 & 72,356 & $3.56 \mathrm{e}+07$ & 668 & 668 & 0x1.77b31324422c1p-38 & 0x1.5a2eda56201fbp-38 & $4.36 \mathrm{e}-02$ & $9.92 \mathrm{e}-02$ & $6.02 \mathrm{e}-01$ \\
\hline 494_bus & 1,666 & $3.89 \mathrm{e}+06$ & 410 & 410 & 0x1.f52f8f $4 c 274 d p-37$ & 0x1.b83293969f70bp-36 & $1.21 \mathrm{e}-02$ & $1.63 \mathrm{e}-02$ & $5.78 \mathrm{e}-02$ \\
\hline $\operatorname{msc04515}$ & 97,707 & $4.78 \mathrm{e}+05$ & 4874 & 4885 & $0 x 1.2 \mathrm{dfe} 5 \mathrm{e} 95 \mathrm{c} 1703 \mathrm{p}-32$ & 0x1.4b0c4d82346dap-32 & $3.26 \mathrm{e}-01$ & $7.56 \mathrm{e}-01$ & $4.80 \mathrm{e}+00$ \\
\hline bcsstk 27 & 56,126 & $1.49 \mathrm{e}+04$ & 331 & 331 & $0 \mathrm{x} 1.2 \mathrm{c} 11 \mathrm{a} 8 \mathrm{f} 939 \mathrm{c} 1 \mathrm{dp}-40$ & 0x1.2b65be5099a69p-40 & $1.45 \mathrm{e}-02$ & $2.53 \mathrm{e}-02$ & $1.04 \mathrm{e}-01$ \\
\hline
\end{tabular}

Table 7. Evaluation of different pure MPI implementations lakymchuk et al. (2019a) of the PCG method using test matrices from the SuiteSparse matrix collection on one Tintorrum's node with $16 \mathrm{MPI}$ processes.

MP I_Reduce and MPI_Bcast, and perform the intra-node DOT product with FPEs. To tackle compiler interference in computations, we reconstruct computations as well as explicitly invoke fma instructions. Both approaches deliver identical results on two clusters to ensure reproducibility of PCG in the number of iterations, the intermediate and final residuals, the direct errors, as well as the vectorsolution on the example of a 3D Poisson's equation with 27 stencil points as well as several test matrices from the SuiteSparse matrix collection. On a single node, the FPEand ExBLAS-based reproducible versions of PCG show the maximum overhead of $2.24 \mathrm{x}$ and $2.66 \mathrm{x}$, respectively, due to 
additional memory allocation and computations. When the communication starts to dominate the execution time, both versions show very low overhead compared with the original non-deterministic implementation: $37.70 \%$ for ExBLAS and $30.50 \%$ for Opt on 768 cores of MareNostrum4; $5.6 \%$ for ExBLAS and $4.6 \%$ for Opt on 128 cores of Tintorrum. This is a solid argument in favor of the reproducible PCG at scale. The code is available at https://github.com/ riakymch/ReproCG_MPI_OMP.

Our study promotes the adoption of reproducibility by design through the proper choice of the underlying libraries as well as a moderate programmability effort. For instance, a brief guidance would be 1) for fundamental numerical computations, to leverage reproducible underlying libraries such as ExBLAS, ReproBLAS, or OzBLAS; and 2) analyze the algorithm and make it reproducible through eliminating any uncertainties that may violate associativity such as reductions and use/ non-use of fmas. Additionally, we argue the need for the bit-wise reproducible and correctly-rounded results for iterative solvers as, nevertheless, they will be enhanced during subsequent iterations as we do not reach the desired tolerance and, thus, do not exploit at full the obtained bit-wise results.

Our future work aims to conduct a deeper analysis of the lightweight approach to support our experimental results. One idea is to bind the length of FPEs to the condition number of the input problem and/or its dynamic range similarly to Carson and Higham (2018) for the mixedprecision direct linear solver.

\section{Acknowledgements}

To begin with, we would like to thank the reviewers for their valuable comments and suggestions. This research was partially supported by the European Union's Horizon 2020 research, innovation programme under the Marie Skłodowska-Curie grant agreement via the Robust project No. 842528 as well as the Project HPC-EUROPA3 (INFRAIA-2016-1-730897), with the support of the H2020 EC RIA Programme; in particular, the author gratefully acknowledges the support of Vicenç Beltran and the computer resources and technical support provided by BSC. The researchers from Universitat Jaume I (UJI) and Universitat Politécnica de Valencia (UPV) were supported by MINECO project TIN201782972-R. Maria Barreda was also supported by the POSDOCA/2017/11 project from the Universitat Jaume I.

\section{References}

Aliaga JI, Barreda M, Flegar G, Bollhöfer M and Quintana-Ortí ES (2017) Communication in task-parallel ILU-preconditioned CG solvers using MPI+OmpSs. Concurrency and Computation: Practice and Experience 29(21): e4280.

Bailey DH (2013) High-precision computation: applications and challenges. In: Proceedings of ARITH-21. IEEE, p. 1. Keynote talk.

Barreda M, Aliaga J, Beltran V and Casas M (2019) Iterationfusing conjugate gradient for sparse linear systems with mpi + ompss. Journal of Supercomputing DOI:10. 1051/proc/201445023. URL https://doi:10.1007/ s11227-019-03100-4.

Barrett R, Berry M, Chan TF, Demmel J, Donato J, Dongarra J, Eijkhout V, Pozo R, Romine C and der Vorst HV (1994)
Templates for the Solution of Linear Systems: Building Blocks for Iterative Methods, 2nd Edition. SIAM.

Burgess N, Goodyer C, Lutz DR and Hinds CN (2019) Highprecision anchored accumulators for reproducible floatingpoint summation. IEEE Transactions on Computers 68(7): 967-978.

Carson E and Higham NJ (2018) Accelerating the solution of linear systems by iterative refinement in three precisions. SIAM J. Sci. Comput. 40(2): A817-A847. DOI:10.1137/17M1140819.

Collange S, Defour D, Graillat S and Iakymchuk R (2015) Numerical reproducibility for the parallel reduction on multiand many-core architectures. ParCo 49: 83-97.

Dekker TJ (1971) A floating point technique for extending the available precision. Numerische Mathematik 18(3): 224-242.

Demmel J and Nguyen HD (2013) Fast reproducible floating-point summation. In: Proceedings of ARITH-21. pp. 163-172.

Demmel J and Nguyen HD (2015) Parallel Reproducible Summation. IEEE Transactions on Computers 64(7): 20602070.

Dongarra JJ, Croz JD, Hammarling S and Duff I (1990) A set of level 3 basic linear algebra subprograms. ACM TOMS 16(1): $1-17$.

forum M (2019) MPI forum. http: / / www . mpi-forum. org.

Fousse L, Hanrot G, Lefèvre V, Pélissier P and Zimmermann P (2007) MPFR: A Multiple-precision Binary Floating-point Library with Correct Rounding. ACM TOMS 33(2): 13. DOI: 10.1145/1236463.1236468.

Golub GH and Loan CFV (2013) Matrix Computations. 4th edition. Baltimore: The Johns Hopkins University Press.

Gropp W, Hoefler T, Thakur R and Lusk E (2014) Using advanced MPI: Modern features of the message-passing interface. MIT Press.

Hida Y, Li XS and Bailey DH (2001) Algorithms for quad-double precision floating point arithmetic. In: Proceedings of ARITH15. pp. 155-162. DOI:10.1109/ARITH.2001.930115.

Hunold S and Carpen-Amarie A (2016) Reproducible MPI benchmarking is still not as easy as you think. IEEE Transactions on Parallel and Distributed Systems 27(12): 3617-3630. DOI:10.1109/TPDS.2016.2539167.

Iakymchuk R, Barreda M, Wiesenberger M, Aliaga JI and Quintana-Orti ES (2019a) Reproducibility Strategies for Parallel Preconditioned Conjugate Gradient. JCAM Available online 2nd January 2020. DOI: 10.1016/j.cam.2019.112697. HAL preprint: hal-02391618.

Iakymchuk R, Collange S, Defour D and Graillat S (2015) ExBLAS: Reproducible and accurate BLAS library. In: Proceedings of the NRE2015 workshop held as part of SC15. Austin, TX, USA, November 15-20, 2015.

Iakymchuk R, Collange S, Defour D and Graillat S (2017) ExBLAS (Exact BLAS) library. Available on the WWW, https: //exblas.lip6.fr/. Accessed 31-JAN-2019.

Iakymchuk R, Defour D, Collange S and Graillat S (2016) Reproducible and Accurate Matrix Multiplication. Springer LNCS 9553: 126-137.

Iakymchuk R, Graillat S, Defour D and Quintana-Orti ES (2019b) Hierarchical Approach for Deriving a Reproducible Unblocked LU factorization. IJHPCA 33(5): 791-803. HAL preprint: hal01419813. 
IEEE Computer Society (2008) IEEE Standard for Floating-Point Arithmetic. IEEE Standard 754-2008.

Imamura T, Mukunoki D, Iakymchuk R, Jézéquel $\mathrm{F}$ and Graillat $\mathrm{S}$ (2019) Numerical reproducibility based on minimal-precision validation. In: the CRE2019 workshop held as part of SC19. Denver, Co, USA, November 17-22, 2019.

Knuth DE (1969) The Art of Computer Programming: Seminumerical Algorithms, volume 2. Addison-Wesley.

Kulisch U and Snyder V (2011) The Exact Dot Product As Basic Tool for Long Interval Arithmetic. Computing 91(3): 307-313.

Kulisch UW (2013) Computer arithmetic and validity, de Gruyter Studies in Mathematics, volume 33. 2nd edition. Berlin: Walter de Gruyter \& Co. Theory, implementation, and applications.

Lawson CL, Hanson RJ, Kincaid DR and Krogh FT (1979) Basic linear algebra subprograms for Fortran usage. ACM TOMS 5(3): 308-323.

Lutz DR and Hinds CN (2017) High-precision anchored accumulators for reproducible floating-point summation. In: Proceedings of ARITH-24. IEEE, London, UK, pp. 98-105.

Mukunoki D, Ogita T and Ozaki K (2020) Accurate and reproducible blas routines with ozaki scheme for many-core architectures. In: Proc. International Conference on Parallel Processing and Applied Mathematics (PPAM2019). Lecture Notes in Computer Science, volume 12043. pp. 516-527.

Nguyen HD and Demmel J (2015) Reproducible tall-skinny QR. In: Proceedings of ARITH-22. pp. 152-159. DOI:10.1109/ARITH. 2015.28.

Ogita T, Rump SM and Oishi S (2005) Accurate sum and dot product. SIAM J. Sci. Comput 26: 1955-1988.

OpenMP ARB (2019) The OpenMP API specification for parallel programming. https: //www. openmp.org/.

Ozaki K, Ogita T, Oishi S and Rump SM (2012) Error-free transformations of matrix multiplication by using fast routines of matrix multiplication and its applications. Numerical Algorithms 59(1): 95-118.

Priest DM (1991) Algorithms for arbitrary precision floating point arithmetic. In: 10th IEEE Symposium on Computer Arithmetic. IEEE, pp. 132-143.

Rump SM, Ogita T and Oishi S (2008a) Accurate floating-point summation part i: Faithful rounding. SIAM J. Sci. Comput. 31(1): 189-224. DOI:10.1137/050645671. URL https: //doi.org/10.1137/050645671.

Rump SM, Ogita T and Oishi S (2008b) Accurate floating-point summation part ii: Sign, k-fold faithful and rounding to nearest. SIAM J. Sci. Comput. 31(2): 1269-1302.

Rump SM, Ogita T and Oishi S (2010) Fast high precision summation. Nonlinear Theory and Its Applications, IEICE 1(1): 2-24.

Saad Y (2003) Iterative methods for sparse linear systems. 3rd edition. Philadelphia, PA, USA: SIAM.

Wiesenberger M, Einkemmer L, Held M, Gutierrez-Milla A, Xavier Saez X and Iakymchuk R (2019) Reproducibility, accuracy and performance of the feltor code and library on parallel computer architectures. Computer Physics Communications 238: 145-156.

Zielke G and Drygalla V (2003) Genaue lösung linearer gleichungssysteme. GAMM Mitt. Ges. Angew. Math. Mech. 26: $7-107$. 